\title{
A flexible fuzzy regression method for addressing nonlinear uncertainty on aesthetic quality assessments
}

\author{
Kit Yan Chan, Hak Keung Lam, Senior Member, IEEE, Cedric Ka Fai Yiu, Tharam S. Dillon, \\ Life Fellow IEEE
}

\begin{abstract}
Development of new products or services requires knowledge and understanding of aesthetic qualities that correlate to perceptual pleasure. As it is not practical to develop a survey to assess aesthetic quality for all objective features of a new product or service, it is necessary to develop a model to predict aesthetic qualities. In this paper, a fuzzy regression method is proposed to predict aesthetic quality from a given set of objective features and to account for uncertainty in human assessment. The proposed method overcomes the shortcoming of statistical regression, which can predict only quality magnitudes but cannot predict quality uncertainty. The proposed method also attempts to improve traditional fuzzy regressions, which simulate a single characteristic with which the estimated uncertainty can only increase with the increasing magnitudes of objective features. The proposed fuzzy regression method uses genetic programming (GP) to develop nonlinear structures of the models, and model coefficients are determined by optimizing the fuzzy criteria. Hence the developed model can be used to fit the nonlinearities of sample magnitudes and uncertainties. The effectiveness and the performance of the proposed method are evaluated by the case study of perceptual images, which are involved with different sampling natures and with different amounts of samples. This case study attempts to address different characteristics of human assessments. The outcomes demonstrate that more robust models can be developed by the proposed fuzzy regression method compared with the recently developed fuzzy regression methods, when the model characteristics and fuzzy criteria are taken into account.

Index Terms - aesthetic quality assessment/prediction, fuzzy regression, perceptual imaging, uncertainty estimates
\end{abstract}

\section{INTRODUCTION}

A esthetic quality evaluates the nature of art, beauty, and taste in relation to the creation and appreciation of products [1]. It is essential to take aesthetic quality into account when developing pleasurable products. Better aesthetic quality increases products' appeal to potential buyers and produces a more harmonious product [70]. For example, two teapots with the same capacity and size are used in a café - one with an attractive colour and shape, the other with unattractive ones [48]. Despite the fact that the teapots have

Manuscript received $\mathrm{xxxx}$; accepted $\mathrm{xxxx}$. Date of publication $\mathrm{xxxx}$; date of current version $\mathrm{xxxx}$

Kit Yan Chan is with The Department of Electrical and Computer Engineering, Curtin University, Australia (e-mail: kit.chan@,curtin.edu.au).

Hak Keung Lam is with the Department of Informatics, King's College London, United Kingdom (e-mail: hak-keung.lam@kcl.ac.uk).

Cedric Ka Fai Yiu is with the Department of Applied Mathematics, The Hong Kong Polytechnic University, Hong Kong (macyiu@polyu.edu.hk)

Tharam S. Dillon is with the Department of Computer Science and IT, Latrobe University, Melbourne, Australia (e-mail: tharam.dillon7@gmail.com). the same practical features, people find it more enjoyable to drink from the attractive one.

To assess aesthetic qualities, two types of approaches are generally used: The first approach uses survey-based questionnaires to obtain aesthetic scores from a product. Conducting a full survey requires significant amount of time and cost to assess all objective features of a product or service. Also the survey cannot be implemented in real time. The second approach assumes that human perceptions of aesthetic qualities are trigger by objective features like colours and object configurations [70]. Aesthetic quality models can be developed to predict aesthetic qualities by correlating with objective features. Statistical regression has commonly been used for developing aesthetic quality models to predict aesthetic quality for plastic surgery [14], restorative material for teeth [44], structural landscape design [71], visuals of images/video [41,58], and website design [52,29]. The statistical regressions are commonly used because statistical regression models are explicit. Based on the models, ones can analyze significances and interactions of objective features and also ones can compute confidence intervals for assessing the aesthetic quality. Comparing with artificial neural networks [51] and fuzzy T-S systems [34,35], they have more transparency than artificial neural networks [60].

However, human assessments of aesthetic qualities are subjective and are therefore inherently difficult to measure. Although the statistical regression can be used to estimate magnitudes of aesthetic qualities, the perceived uncertainties caused by human assessments cannot be addressed. To overcome this shortcoming, fuzzy regression [57] has been developed to address uncertainties caused by human assessments of customer satisfaction [24,38,53], affective quality [56], image quality [5,9] and option prices in finance [45]. Similar to statistical regression models, fuzzy regression models are structured by polynomials. However, statistical regression models consist only of coefficients in real numbers and fuzzy regression models consist of fuzzy coefficients in fuzzy numbers. Fuzzy coefficients attempt to map objective features to aesthetic quality which is in the domain of fuzzy numbers [27]. Perceived uncertainty caused by human assessments can be addressed, when the aesthetic quality in fuzzy numbers is estimated.

A potential shortcoming was ascertained in using fuzzy regression to address uncertainties in human assessments [25]. These fuzzy regression models $[4,8,15,16,18,19,20,21,24,37,38$, $42,45,47,49,54,55,64,66]$ address only one characteristic that the estimated uncertainty of human assessments can only increase with 
the increasing magnitudes of objective features. The assumption may suit instrumentation measurements because the amount of uncertainty increases when measurement magnitudes increase [43]. However, the uncertainty of assessing aesthetic quality can be smaller or unchanged when feature magnitudes are increased [25]. For example, when aesthetic qualities of images are evaluated, we may have less uncertainty in evaluating images with high or low qualities than those with medium qualities [13]. Hence more uncertainty is generated when images with medium qualities are evaluated. Several approaches have been proposed to address this shortcoming of estimating uncertainty $[25,46,62]$. Analytical study demonstrates that those methods still cannot address decreasing or statics uncertainty trends when magnitudes of objective features are increasing [39].

To overcome this shortcoming, this paper proposes a novel fuzzy regression namely NON-SC-FR, which attempts to generate aesthetic quality models for addressing nonlinear uncertainty. The NON-SC-FR uses a commonly used evolutionary computational approach, GP [17,21,36], to develop the model structures for the magnitudes and uncertainties of aesthetic qualities. The model structures are correlated with object features and aesthetic qualities. GP is incorporated with NON-SC-FR as GP is effective to generate polynomial models for a complex and highly nonlinear systems [31]. Also, GP is effective in modeling nonlinear quantities and uncertainties. After the model structures are determined, the model coefficients are computed by optimizing the fuzzy criteria [3,37] which are commonly used to fit uncertain samples for both fuzzy numbers and real numbers.

The effectiveness and the performance of the proposed NONSC-FR are evaluated by two databases for image quality assessment (IQA) namely TID [50] and VLC [69] databases, where the IQA is important for image compression, transmission and reconstruction [12]. The IQA is used for evaluating the NON-SC-FR as IQA is involved with nonlinear magnitudes and uncertainties [13]. Also, TID [50] and VLC [69] databases contain samples with real and fuzzy numbers respectively. Hence the effectiveness of the NONSC-FR can be evaluated for modelling both sample types. TID and VLC contain 2125 and 552 samples respectively. Different characteristics of human assessments can be addressed, as different amount of samples are considered. We compare the proposed NON-SC-FR with four existing fuzzy regressions [4,20,39,61], which have been recently developed for overcoming the aforementioned deficiency of uncertainty estimation. These two case studies of IQA verify that the proposed NON-SC-FR is able to develop better models which can achieve four better fuzzy criteria, confidence index [63], fuzzy credibility [37], overall fuzzy spread [57] and fuzzy number distance [3].

The contents of the paper are summarized as:

1. Section II discusses the formulation and mechanism of aesthetic quality models which correlate aesthetic quality with objective features. An example of IQA is given to discuss the common shortcoming of the existing approaches of fuzzy regression which cannot address nonlinear uncertainties of assessing aesthetic quality. It gives the motivation of why we propose the NON-SC-FR in order to model the nonlinear uncertainties.

2. Section III discusses the operations and mechanisms of the proposed NON-SC-FR, which attempts to overcome this common limitation. The NON-SC-FR attempts to model the magnitude and uncertainty of aesthetic quality which can be both nonlinear. The NON-SC-FR can be used to develop models when the data in either fuzzy or real numbers is given. The real numbers simulate that the magnitudes of aesthetic quality are only available. The fuzzy numbers simulate that the variances and magnitudes of aesthetic quality are both available.

3. Section IV presents two case studies of IQA which involve data in fuzzy and real numbers. These two case studies verify that the proposed NON-SC-FR outperforms four existing methods which are developed for overcoming the limitation of classical fuzzy regressions. Also the characteristics of the NON-SC-FR and the tested methods are discussed. Section V gives a conclusion and it also discusses the future study for this research.

\section{FUZZY REGRESSION FOR AESTHETIC QUALITY ESTIMATION}

People generally assess aesthetic qualities based on human emotional or perceptual judgment [70]. Aesthetic qualities are evaluated around an integer with some degree of uncertainty [68]. For example, in IQA, ones may use a 5-point scale to score the image quality. An image with 'high' quality would be scored with a linguistic term 'about 5'; an image with 'low' quality would be scored with 'about 1'; the one with 'fair' quality would be scored with 'about 3'. The linguistic term 'about $y^{c}$ ' can be considered as a fuzzy number, $\tilde{y}=\left(y^{c}, y^{l}, y^{r}\right)$, where $\tilde{y}$ is engaged with the membership [68] given by (1).

$$
\mu_{\tilde{y}}(y)=\left\{\begin{array}{ll}
1 & y=y^{c} \\
1-\frac{y^{c}-y}{y^{l}} & y^{c}-y^{l} \leq y<y^{c} \\
1-\frac{y-y^{c}}{y^{r}} & y^{c}<y \leq y^{c}+y^{r} \\
0 & \text { otherwise }
\end{array},\right.
$$

where $y^{c}$ is a real number. It denotes the magnitude of the aesthetic quality; the uncertainty of the assessment is indicated by the sum of $y^{l}$ and $y^{r}$ with $y^{l}, y^{r} \geq 0$.

To predict the aesthetic qualities in fuzzy numbers, the following aesthetic quality model, $\mathfrak{I}_{\mathrm{PQM}}(\bar{x})$, can be used:

$$
\tilde{y}=\left(y^{c}, y^{l}, y^{r}\right)=\mathfrak{J}_{P Q M}(\bar{x})=\left(\varphi_{P Q M}^{c}(\bar{x}), \varphi_{P Q M}^{l}(\bar{x}), \varphi_{P Q M}^{r}(\bar{x})\right)
$$

where $\bar{x}=\left(x_{1}, x_{2}, \ldots, x_{m}\right) ; \quad x_{j}$ denotes the $j$-th objective feature with $j=1,2, \ldots, m ; m$ is the number of $x_{j} ; \mathfrak{J}_{P Q M}(\bar{x})$ is the functional relationship which correlates $\bar{x}$ to $y ; \varphi_{\mathrm{PQM}}^{c}(\bar{x})$, $\varphi_{P Q M}^{l}(\bar{x})$ and $\varphi_{P Q M}^{r}(\bar{x})$ correlate $\bar{x}$ to $y^{c}, y^{l}$ and $y^{r}$ respectively.

The estimated uncertainty, $\Phi\left(\mathfrak{I}_{P Q M}(\bar{x})\right)$, with respect to $\bar{x}$ is defined by [50]:

$$
\Phi\left(\mathfrak{I}_{P Q M}(\bar{x})\right)=\varphi_{P Q M}^{l}(\bar{x})+\varphi_{P Q M}^{r}(\bar{x})
$$

\section{A. Fuzzy linear and nonlinear regressions}

$\mathfrak{I}_{P Q M}$ can be developed based on fuzzy linear regression [49], $F_{P Q M}^{L i n}$, which is given as:

$$
\tilde{y}=\left(y^{c}, y^{l}, y^{r}\right)=F_{P Q M}^{L i n}(\bar{x})=\left(a_{0}^{c}, a_{0}^{l}, a_{0}^{r}\right)+\sum_{i=1}^{m}\left(a_{i}^{c}, a_{i}^{l}, a_{i}^{r}\right) \cdot x_{i}
$$


where $\left(a_{i}^{c}, a_{i}^{l}, a_{i}^{r}\right)$ are the fuzzy coefficients; $a_{i}^{c}, a_{i}^{l}$ and $a_{i}^{r}$ are the central, left and right spreads of the fuzzy coefficient respectively. $F_{P Q M}^{L i n}(\bar{x})$ in (4) can be rewritten as:

$$
F_{P Q M}^{L i n}(\bar{x})=\left(f_{\text {Lin }}^{c}(\bar{x}), f_{\text {Lin }}^{l}(\bar{x}), f_{\text {Lin }}^{r}(\bar{x})\right)
$$

where:

$$
\begin{aligned}
& f_{\text {Lin }}^{c}(\bar{x})=a_{0}^{c}+a_{1}^{c} x_{1}+a_{2}^{c} x_{j}+\cdots+a_{m}^{c} x_{m} ; \\
& f_{\text {Lin }}^{l}(\bar{x})=a_{0}^{l}+a_{1}^{l} x_{1}+a_{2}^{l} x_{j}+\cdots+a_{m}^{l} x_{m} ; \\
& f_{\text {Lin }}^{r}(\bar{x})=a_{0}^{r}+a_{1}^{r} x_{1}+a_{2}^{r} x_{j}+\cdots+a_{m}^{r} x_{m} ;
\end{aligned}
$$

$f_{\text {Lin }}^{c}(\bar{x})$ is the central function of $F_{P Q M}^{\text {Lin }}(\bar{x})$ which is corresponding to $y^{c} ; f_{\text {Lin }}^{l}(\bar{x})$ and $f_{\text {Lin }}^{r}(\bar{x})$ are the left and right spread functions of $F_{P Q M}^{N o n}(\bar{x})$ which correspond to $y^{l}$ and $y^{r}$ respectively. The estimated uncertainty for $F_{P Q M}^{\operatorname{Lin}}(\bar{x})$ is given as:

$$
\Phi\left(F_{P Q M}^{\text {Lin }}(\bar{x})\right)=f_{\text {Lin }}^{l}(\bar{x})+f_{\text {Lin }}^{r}(\bar{x})
$$

As $F_{P Q M}^{\text {Lin }}(\bar{x})$ is formulated by a linear polynomial, it is ineffective to fit nonlinear samples. Therefore, $\mathfrak{I}_{\mathrm{PQM}}(\bar{x})$ can be developed by the fuzzy nonlinear regression, $F_{P Q M}^{N o n}(\bar{x})$, which is in a Kolmogorov-Gabor polynomial form as shown in (6) [2].

$$
\begin{aligned}
\tilde{y}=\left(y^{c},\right. & \left.y^{l}, y^{r}\right)=F_{P Q M}^{N o n}(\bar{x})=\left(b_{0}^{c}, b_{0}^{l}, b_{0}^{r}\right)+\sum_{i_{1}=1}^{m}\left(b_{i_{1}}^{c}, b_{i_{1}}^{l}, b_{i_{1}}^{r}\right) \cdot x_{i_{1}} \\
& +\sum_{i_{1}=1}^{m} \sum_{i_{2}=1}^{m}\left(b_{i_{1}, i_{2}}^{c}, b_{i_{1}, i_{2}}^{l}, b_{i_{1}, i_{2}}^{r}\right) \cdot x_{i_{1}} \cdot x_{i_{2}} \\
& +\sum_{i_{1}=1}^{m} \sum_{i_{2}=1}^{m} \sum_{i_{3}=1}^{m}\left(b_{i_{1}, i_{2}, i_{3}}^{c}, b_{i_{1}, i_{2}, i_{3}}^{l}, b_{i_{1}, i_{2}, i_{3}}^{r}\right) \cdot x_{i_{1}} \cdot x_{i_{2}} \cdot x_{i_{3}} \\
& +\ldots+\sum_{i_{1}=1}^{m} \ldots \sum_{i_{d}=1}^{m}\left(b_{i_{1}, i_{2}, \ldots i_{d}}^{c}, b_{i_{1}, i_{2}, \ldots i_{d}}^{l}, b_{i_{1}, i_{2}, \ldots i_{d}}^{r}\right) \prod_{j=1}^{3<d<m} x_{j} \\
& +\ldots+\left(b_{1,2, \ldots, m}^{c}, b_{1,2, \ldots, m}^{l}, b_{1,2, \ldots, m}^{r}\right) \cdot x_{1} \cdot x_{2} \cdot \ldots \cdot x_{m}
\end{aligned}
$$

where $\left(b_{*}^{c}, b_{*}^{l}, b_{*}^{r}\right)$ are the fuzzy coefficients with any combination '*'; $b_{*}^{c}$ is the central; $b_{*}^{l}$ and $b_{*}^{r}$ represent the left and right spreads respectively. $F_{P Q M}^{N o n}(\bar{x})$ in (6) can be rewritten as:

$$
F_{P Q M}^{N o n}(\bar{x})=\left(f_{\text {Non }}^{c}(\bar{x}), f_{\text {Non }}^{l}(\bar{x}), f_{\text {Non }}^{r}(\bar{x})\right),
$$

where

$$
\begin{aligned}
& f_{\text {Non }}^{\gamma}(\bar{x})=b_{0}^{\gamma}+\sum_{i_{1}=1}^{m} b_{i_{1}}^{\gamma} \cdot x_{i_{1}}+\sum_{i_{1}=1}^{m} \sum_{i_{2}=1}^{m} b_{i_{1}, i_{2}}^{\gamma} \cdot x_{i_{1}} \cdot x_{i_{2}}+ \\
& \sum_{i_{1}=1}^{m} \sum_{i_{2}=1}^{m} \sum_{i 3=1}^{m} b_{i_{1}, i_{2}, i_{3}}^{\gamma} \cdot x_{i_{1}} \cdot x_{i_{2}} \cdot x_{i_{3}}+\ldots+\sum_{i_{1}=1}^{m} \ldots \sum_{i_{d}=1}^{m} b_{i_{1}, i_{2}, . . i_{d}}^{\gamma} \prod_{j=1}^{3<d<m} x_{j} ; \\
& \quad+. .+b_{1,2, . ., m}^{\gamma} \cdot x_{1} \cdot x_{2} \cdot \ldots \cdot x_{m}
\end{aligned}
$$

with $\gamma \in\left\{c^{\prime},{ }^{\prime} l l^{\prime},{ }^{\prime} r^{\prime}\right\}$. In $F_{P Q M}^{\text {Non }}(\bar{x}), f_{\text {Lin }}^{c}(\bar{x})$ is the central function which corresponds to $y^{c} ; f_{\text {Lin }}^{l}(\bar{x})$ and $f_{\text {Lin }}^{r}(\bar{x})$ are the left and right spread functions which correspond to $y^{c}$ and $y^{r}$ respectively. The estimated fuzziness for $F_{P Q M}^{N o n}(\bar{x})$ is given as:

$$
\Phi\left(F_{P Q M}^{\text {Non }}(\bar{x})\right)=f_{\text {Lin }}^{l}(\bar{x})+f_{\text {Lin }}^{r}(\bar{x})
$$

Figures 1a and $1 \mathrm{~b}$ illustrate $F_{P Q M}^{\text {Lin }}(\bar{x})$ and $F_{P Q M}^{\text {Non }}(\bar{x})$ respectively. They illustrate the central functions $f_{\text {Lin }}^{c}(\bar{x})$ and $f_{\text {Non }}^{c}(\bar{x})$, and the spread functions, $f_{\text {Lin }}^{l}(\bar{x}), f_{\text {Lin }}^{r}(\bar{x}), f_{\text {Non }}^{l}(\bar{x})$ and $f_{\text {Non }}^{r}(\bar{x})$, with respect to $\bar{x}$. The estimated uncertainties, $\Phi\left(F_{P Q M}^{L i n}(\bar{x})\right)$ in $(5 \mathrm{e})$ and
$\Phi\left(F_{P Q M}^{N o n}(\bar{x})\right)$ in $(7 \mathrm{e})$, are also illustrated in Figures $1 \mathrm{a}$ and $1 \mathrm{~b}$ respectively.

In this research, we consider the case that the uncertainty is related to the independent variables, $\bar{x}$ and it is also related to the constants $a_{0}^{r}$ and $a_{0}^{l}$. In some cases, the uncertainty of instrumentation measurements increases when the magnitude of $\bar{x}$ increases [42]. Hence, $\Phi\left(F_{P Q M}^{\operatorname{Lin}}(\bar{x})\right)$ is suitable on estimating the uncertainty of instrumentation measurements, when the measurement uncertainty is linearly related to $\bar{x} . a_{0}^{r}$ and $a_{0}^{l}$ can be used to covered some uncertainty which are not correlated with $\bar{x}$. $\Phi\left(F_{P Q M}^{\text {Non }}(\bar{x})\right)$ is more suitable on estimating uncertainty of assessing aesthetic quality, as the uncertainty may not be increased with the increasing magnitude of $\bar{x}$. This may not be always the case that uncertainty is increased when the magnitude of $\bar{x}$ increases. For example, when aesthetic qualities of images are assessed, ones may have less uncertainty or more confidence in evaluating very high or very low qualities than that in evaluating medium qualities [25]. Therefore, the amount of uncertainty of assessing aesthetic quality may be nonlinearly correlated to $\bar{x}$.

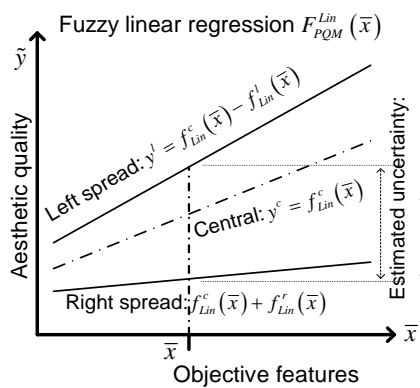

Fig. 1a: Fuzzy linear regression $F_{P Q M}^{L i n}(\bar{x})$

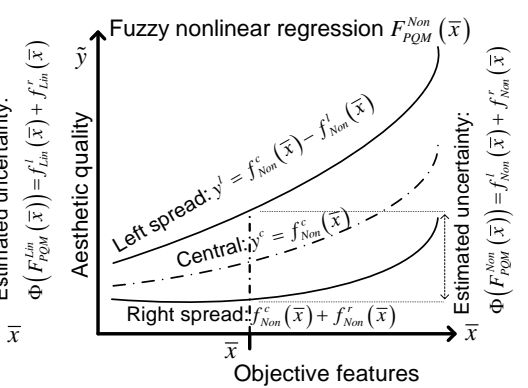

Fig. 1b: Fuzzy nonlinear regression $F_{P Q M}^{\text {Non }}(\bar{x})$

\section{B. Common deficiency of fuzzy regression}

For both $F_{P Q M}^{L i n}(\bar{x})$ in (4) and $F_{P Q M}^{N o n}(\bar{x})$ in (6), the estimated uncertainty is indicated by the sum of left and right spreads given in (5e) and (7c) respectively. Uncertainty cannot be a negative value. Therefore, in order to ensure positive uncertainties, triangular fuzzy numbers are generally used as the fuzzy coefficients in $F_{P Q M}^{L i n}[8,37$, $15,16,19,23,37,42,49,47,54,55,64]$ and in $F_{P Q M}^{\text {Non }}[4,24,20,45,66]$. The left spreads (i.e. $a_{i}^{l}$ and $b_{*}^{l}$ ) and the right spreads (i.e. $a_{i}^{r}$ and $b_{*}^{r}$ ) of the triangular fuzzy numbers are constrained as positive values. When the magnitudes of $\bar{x}$ increase, the magnitudes of the spread functions, $f_{\text {Lin }}^{l}(\bar{x}), f_{\text {Lin }}^{r}(\bar{x}), f_{\text {Non }}^{l}(\bar{x})$ and $f_{\text {Non }}^{r}(\bar{x})$, also increase. Hence, the estimated uncertainties, $\Phi\left(F_{P Q M}^{\operatorname{Lin}}(\bar{x})\right)$ and $\Phi\left(F_{P Q M}^{N o n}(\bar{x})\right)$, also increase as $a_{i}^{l}, b_{*}^{l}, a_{i}^{r}$ and $b_{*}^{r}$ are all positive. We consider the simple models, $F_{P Q M}^{L i n}\left(\left[x_{1}\right]\right)$ and $F_{P Q M}^{N o n}\left(\left[x_{1}\right]\right)$, with one objective feature, $x_{1}$, respectively as:

$$
\begin{aligned}
F_{P Q M}^{L i n}\left(\left[x_{1}\right]\right) & =\left(a_{0}^{c}+a_{1}^{c} x_{1}, a_{0}^{l}+a_{1}^{l} x_{1}, a_{0}^{r}+a_{1}^{r} x_{1}\right) \\
F_{P Q M}^{N o n}\left(\left[x_{1}\right]\right)= & \left(b_{0}^{c}+b_{1}^{c} \cdot x_{1}+b_{1,1}^{c} \cdot x_{1}^{2}, b_{0}^{l}+b_{1}^{l} \cdot x_{1}+b_{1,1}^{l} \cdot x_{1}^{2},\right. \\
& \left.b_{0}^{r}+b_{1}^{r} \cdot x_{1}+b_{1,1}^{r} \cdot x_{1}^{2}\right)
\end{aligned}
$$




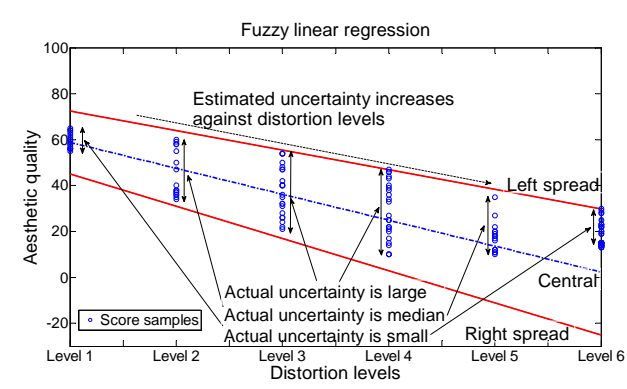

Figure 2a: Fuzzy linear regression $F_{P Q M}^{L i n}\left(\left[x_{1}\right]\right)$

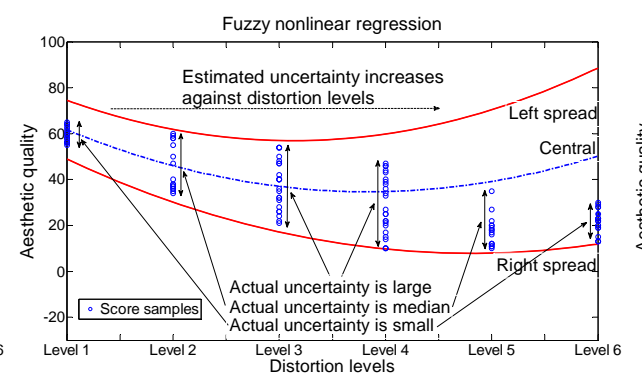

Figure 2b: Fuzzy nonlinear regression $F_{P Q M}^{N o n}\left(\left[x_{1}\right]\right)$

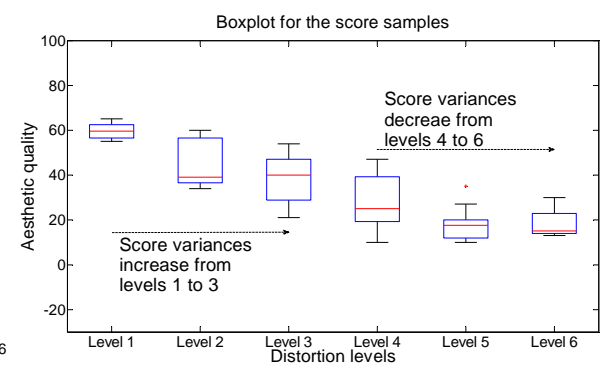

Figure 2c: Boxplot for score samples

Table 1: Contaminated images with 6 distortion levels

\begin{tabular}{|c|c|c|c|c|c|c|}
\hline Distortion & Level 1 & Level 2 & Level 3 & Level 4 & Level 5 & Level 6 \\
\hline Mean scores & 59.60 & 44.88 & 37.78 & 28.16 & 19.50 & 18.74 \\
\hline Std scores & 3.26 & 10.07 & 10.59 & 12.43 & 6.66 & 5.36 \\
\hline Score diff. & 10 & 26 & 33 & 37 & 25 & 17 \\
\hline
\end{tabular}

The estimated uncertainties for $F_{P Q M}^{L i n}\left(\left[x_{1}\right]\right)$ and $F_{P Q M}^{N o n}\left(\left[x_{1}\right]\right)$ are given respectively as:

$$
\begin{aligned}
& \Phi\left(F_{P Q M}^{L i n}\left(\left[x_{1}\right]\right)\right)=\left(a_{0}^{l}+a_{1}^{l} x_{1}\right)+\left(a_{0}^{r}+a_{1}^{r} x_{1}\right) \\
& \Phi\left(F_{P Q M}^{L i n}\left(\left[x_{1}\right]\right)\right)=\left(b_{0}^{l}+b_{1}^{l} \cdot x_{1}+b_{1,1}^{l} \cdot x_{1}^{2}\right)+\left(b_{0}^{r}+b_{1}^{r} \cdot x_{1}+b_{1,1}^{r} \cdot x_{1}^{2}\right)
\end{aligned}
$$

(9a) and (9b) show that $\Phi\left(F_{P Q M}^{\text {Lin }}\left(\left[x_{1}\right]\right)\right)$ and $\Phi\left(F_{P Q M}^{N o n}\left(\left[x_{1}\right]\right)\right)$ increase when $x_{1}$ increases. However, it may not be always true that the uncertainty increases linearly with increasing $\bar{x}$ [39].

Here we consider the aesthetic quality estimation for IQA as an example. We used the $19^{\text {th }}$ image in the VCL database [69]. The image was contaminated by Gaussian noise with six distortion levels. $x_{1}$ in (8a) and (9a) are represented as the distortion level. The first row of Table I illustrates six distorted images contaminated with six levels. It shows clearly that the aesthetic quality decreases gradually from level 1 to level 6 . Each distorted image was scored between 18 and 23 times by randomly selecting 118 naive participants who have no experience in perceived imaging. The second row of Table I shows the score means for the six distorted images, which decrease with increasing distortion levels. Table I also shows the score variances, where the third row shows the standard deviation of the scores for each distorted image, and the fourth row shows the scoring differences for each distorted image. They show that the standard deviations and scoring differences increase from levels 1 to 4 , and decrease from levels 4 to 6. Similar characteristics can be found in the boxplot in Figure 2c where the score means decrease, while the score variances increase from levels 1 to 4 and decrease from levels 4 to 6 .

However, the fuzzy regression estimates different uncertainty trends. Based on Hong and Wang's approach [20], the fuzzy coefficients for $F_{P Q M}^{L i n}\left(\left[x_{1}\right]\right)$ and $F_{P Q M}^{N o n}\left(\left[x_{1}\right]\right)$ are found respectively as:

$$
\begin{aligned}
& \left(a_{0}^{c}, a_{0}^{l}, a_{0}^{r}\right)=(58.79,13.75,13.75) ;\left(a_{1}^{c}, a_{1}^{l}, a_{1}^{r}\right)=(-56.47,13.75,13.75) . \\
& \left(b_{0}^{c}, b_{0}^{l}, b_{0}^{r}\right)=(61.68,12.77,12.77) ;\left(b_{1}^{c}, b_{1}^{l}, b_{1}^{r}\right)=(-94.97,12.77,12.77) . \\
& \left(b_{1,1}^{c}, b_{1,1}^{l}, b_{1,1}^{r}\right)=(83.64,12.77,12.77) .
\end{aligned}
$$

Hong and Wang's approach [20] is used here as it was developed to generate both linear and nonlinear fuzzy regression models. The models of $F_{P Q M}^{L i n}\left(\left[x_{1}\right]\right)$ and $F_{P Q M}^{N o n}\left(\left[x_{1}\right]\right)$, are illustrated in Figures $2 \mathrm{a}$ and $2 \mathrm{~b}$ respectively. We can see that the estimated uncertainties increase in both $F_{P Q M}^{L i n}\left(\left[x_{1}\right]\right)$ and $F_{P Q M}^{N o n}\left(\left[x_{1}\right]\right)$ when the distorted levels increase. It does not match the actual uncertainties illustrated in Figures $2 \mathrm{a}$ and $2 \mathrm{~b}$, and it also does not match the characteristics of the actual score variances illustrated in Figure 2c, which increase from levels 1 to 4 and decrease from levels 4 to 6 .

This example illustrates that people may be more uncertain when evaluating median aesthetic quality. People may have more confidence to score images with extreme distortions, when the images either have no distortion or serious distortion. Therefore less uncertainty may be involved for evaluating images contaminated with extreme distortions [13]. This example illustrates the potential deficiency of the fuzzy linear regression that it may not be always true that the estimated uncertainty increases with the increasing objective features. This example shows that fuzzy linear regression may estimate inaccurate uncertainty of aesthetic quality assessment when the certainty is not linearly correlated with the objective features [39].

\section{PROPOSED FUZZY REGRESSION WITH NONLINEAR CENTRAL AND VARYING SPREADS}

To overcome the deficiency of fuzzy regressions discussed in Section II.B, a fuzzy regression, namely NON-SC-FR, is proposed. Based on the aesthetic quality samples, $D_{s}$ in (10), NON-SC-FR can be used to optimize the polynomial structures, $\varphi^{\gamma}$, and polynomial parameters, $\beta^{\gamma}$, with $\gamma \in\left\{c^{\prime},{ }^{\prime} l{ }^{\prime},{ }^{\prime} r^{\prime}\right\}$ which are defined in the aesthetic quality model, $\mathfrak{I}_{P Q M}(\bar{x})$, in (2).

$$
D_{s}=\left\{\tilde{y}_{o}(k), \bar{x}(k) \mid k=1,2, \ldots, N_{D}\right\},
$$

where $\bar{x}(k)=\left\{x_{1}(k), x_{2}(k), \ldots, x_{m}(k)\right\}$ and $\tilde{y}_{o}(k)=\left(y_{o}^{c}(k), y_{o}^{l}(k)\right.$, $\left.y_{o}^{r}(k)\right) ; \tilde{y}_{o}(k)$ can be collected from two data types namely crisp output data [57] and fuzzy output data [3]. For crisp output data, 
only the actual aesthetic qualities in real numbers are captured. Hence, all $y_{o}^{l}(k)=y_{o}^{r}(k)=0$. For fuzzy output data, the uncertainties of the aesthetic qualities are taken into account. Hence, some $y_{o}^{l}(k), y_{o}^{r}(k)>0$.

In NON-SC-FR, GP is employed to generate $\varphi^{\gamma}$ as GP is effective to generate explicit polynomial models for highly nonlinear systems [30]. Hence the models are capable to fit the nonlinearities in sample magnitudes and uncertainties. After $\varphi^{\gamma}$ is determined, $\beta^{\gamma}$ are determined using $D_{s}$. When $D_{s}$ is captured as crisp output data, $\beta^{\gamma}$ attempts to minimize the total fuzziness generated by the model and to cover all samples with a predefined membership level [57]. When $D_{s}$ is captured as the fuzzy one, $\beta^{\gamma}$ attempts to minimize the overall fuzzy distance between estimates and real samples [3].

Figure 3 shows the flow diagram of the NON-SC-FR and the pseudocode of the NON-SC-FR is shown as follows:

\section{Pesudocode of NON-SC-FR}

Input: $D_{s}$; // the aesthetic quality samples in (10)

Output: $\varphi^{\gamma}$ with $\gamma \in\left\{{ }^{\prime} c^{\prime}, l^{\prime},{ }^{\prime}, r^{\prime}\right\}$; //optimal polynomial structures

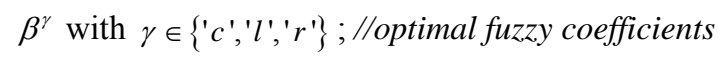

Step 1: Initialize the generation, $t$, by setting $t \leftarrow 0$.

Step 2: Initialize three populations, $\Omega^{\gamma}(t)$ with $\gamma \in\left\{{ }^{\prime} c^{\prime}, l^{\prime},{ }^{\prime} r^{\prime}\right\}$, where each $\Omega^{\gamma}(t)$ consists of $N_{p}$ individuals as,

$$
\Omega^{\gamma}(t)=\left[\theta_{1}^{i}(t), \theta_{2}^{i}(t), \ldots, \theta_{N_{P}}^{i}(t)\right]
$$

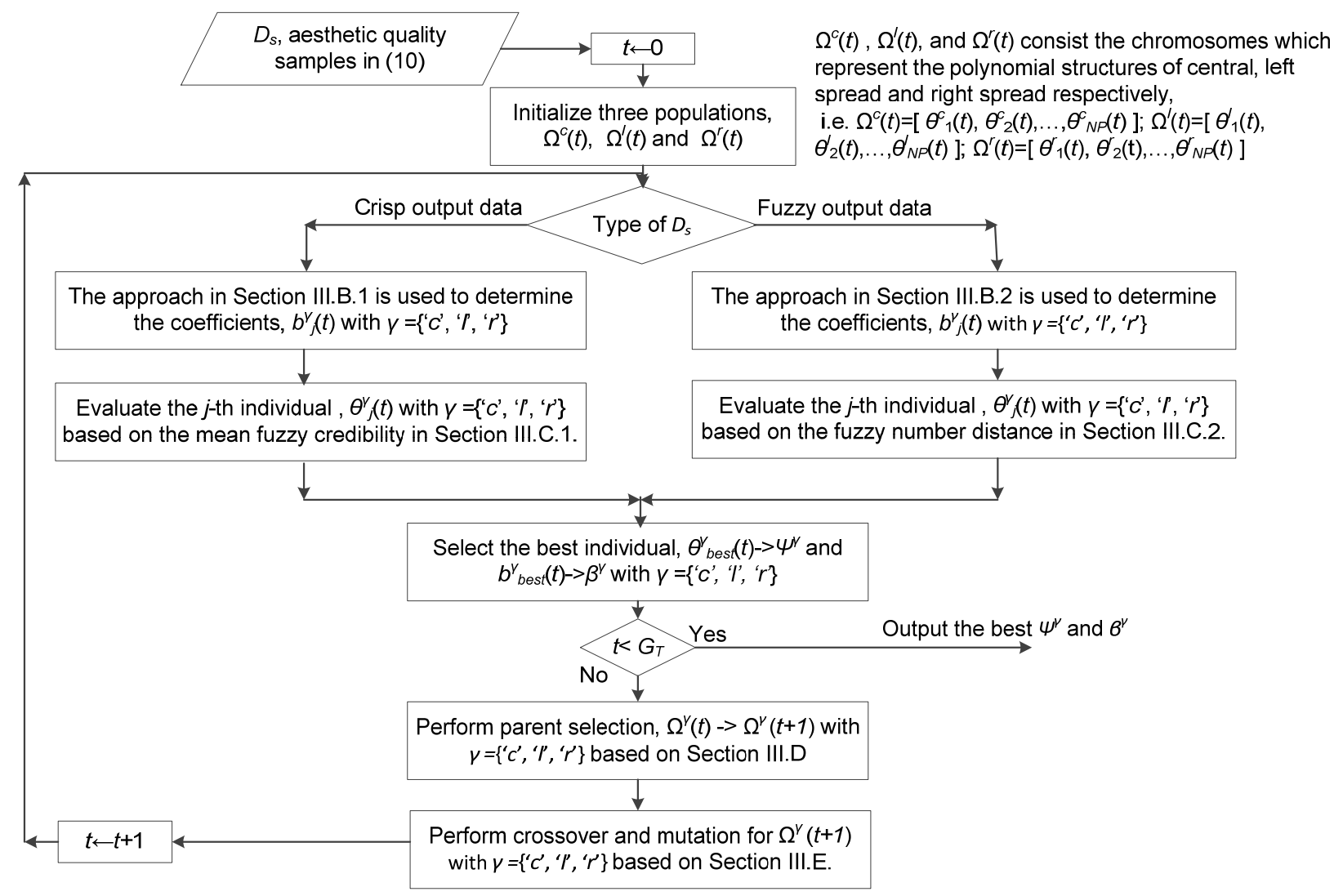

The $j$-th individual, $\theta_{j}^{\gamma}(t)$, represents the polynomial structure with $n_{j}^{\gamma}(t)$ regressors in (12).

Step 3: Determine the fuzzy coefficient vectors, $\bar{b}_{j}^{c}(t), \bar{b}_{j}^{l}(t)$ and $\bar{b}_{j}^{r}(t)$, with respect to $\theta_{j}^{c}(t), \theta_{j}^{l}(t)$ and $\theta_{j}^{r}(t)$ respectively. For crisp output data, the approach in Section III.B. 1 is used. For fuzzy output data, the one in Section III.B.2 is used.

Step 4: Evaluate the fitness of the $j$-th individual which is represented for $\bar{b}_{j}^{\gamma}(t)$ and $\theta_{j}^{\gamma}(t)$ with $\gamma \in\left\{{ }^{\prime} c^{\prime}, l^{\prime},{ }^{\prime} r^{\prime}\right\}$. For crisp output data, mean fuzzy credibility in Section III.C.1 is used. For fuzzy output data, fuzzy number distance in Section III.C.2 is used.

Step 5: Select the best individual with the best polynomial structure, $\theta_{\text {best }}^{\gamma}(t)$, and the best parameter vector, $\bar{b}_{\text {best }}^{\gamma}(t)$, where the (best)-th individual is the best among the $N_{p}$ individuals. Then, put $\theta_{\text {best }}^{\gamma}(t) \rightarrow \varphi^{\gamma}$ and $\bar{b}_{\text {best }}^{\gamma}(t) \rightarrow \beta^{\gamma}$ with $\gamma \in\left\{\right.$ 'c', $\left.^{\prime} l{ }^{\prime},{ }^{\prime} r^{\prime}\right\}$. while $\left(t<G_{T}\right)$ do $\left\{/ / t<G_{T}\right.$ is the termination generation

Step 6: Perform parent selection, $\Omega^{\gamma}(t) \rightarrow \Omega^{\gamma}(t+1)$ with $\gamma \in\left\{{ }^{\prime} c^{\prime}, l^{\prime},{ }^{\prime} r '\right\}$, based on Section III.D.

Step 7: Perform crossover and mutation for $\Omega^{\gamma}(t+1)$ based on Section III.E.

Step 9: Increment $t$ by 1 as $t \rightarrow t+1$.

Step 10: Perform Step 4.

Step 11: Perform Step 5.

Figure 3 Flow diagram for NON-SC-FR 


\section{A. Individual representation}

In NON-SC-FR, the $j$-th individual consists of three polynomial structures, $\theta_{j}^{c}(t), \theta_{j}^{l}(t)$, and $\theta_{j}^{r}(t)$, which are corresponding to the central, $\varphi_{P Q M}^{c}(\bar{x})$, left spread, $\varphi_{P Q M}^{l}(\bar{x})$, and right spread, $\varphi_{\mathrm{PQM}}^{r}(\bar{x})$, of the model, $\mathfrak{I}_{\mathrm{PQM}}(\bar{x})$, in (2). $\theta_{j}^{\gamma}(t)$ with $\gamma \in\left\{{ }^{\prime} c^{\prime}, l^{\prime},{ }^{\prime} r\right\}$ is represented as the hierarchical trees [30], which is efficiency in modelling [65]. In the hierarchical tree, the nonterminal set, $N$, consists of sum operation, ' + ' and multiplication, '.' and the terminal set, $T$, consists of the $m$ objective features, $x_{1}, x_{2}, \ldots, x_{m} . N$ and $T$ are represented as (11a) and (11b) respectively:

$$
\begin{aligned}
& N=\left\{{ }^{\prime}+{ }^{\prime}, \cdot \cdot\right\} ; \\
& T=\left\{x_{1}{ }^{\prime}, x_{2}{ }^{\prime}, \ldots,{ }^{\prime} x_{m}{ }^{\prime}\right\} .
\end{aligned}
$$

$\theta_{j}^{\gamma}(t)$ represents the polynomial structure as,

$$
\theta_{j}^{\gamma}(t)=1+\sum_{i=1}^{n_{j}^{\gamma}(t)} \prod_{q=1}^{r(i)} x_{I_{q}^{\gamma}(i)},
$$

where $n_{j}^{\gamma}(t)$ is the number of regressors in $\theta_{j}^{\gamma}(t) ; r(i)$ is the number of variables at the $i$-th regressor; $I_{q}^{\gamma}(i) \in\{1,2, \ldots, m\}$ with $q=1,2, \ldots, r(i)$ indicates the variable index of the $i$-th regressor; $I_{q}^{\gamma}(i)$ can be represented by the variable index vector, $\bar{I}_{j}^{\gamma}(t)$, as:

$$
\begin{aligned}
& \bar{I}_{j}^{\gamma}(t)=\left[\left(I_{1}^{\gamma}(1), 1\right), \ldots,\left(I_{r(1)}^{\gamma}(1), 1\right),\left(I_{1}^{\gamma}(2), 2\right), \ldots,\left(I_{r(2)}^{\gamma}(2), 2\right),\right. \\
& \left.\quad \ldots,\left(I_{1}^{\gamma}\left(n_{j}^{\gamma}(t)\right), n_{j}^{\gamma}(t)\right), \ldots,\left(I_{r\left(n_{j}^{\gamma}(t)\right)}^{\gamma}\left(n_{j}^{\gamma}(t)\right), n_{j}^{\gamma}(t)\right)\right]
\end{aligned}
$$

where the second coordinate represents the $i$-th regressor. For example, we consider the following polynomial structure with four regressors (i.e. $\left.n_{j}^{\gamma}(t)=4\right)$ :

$$
\theta_{j}^{\gamma}(t)=1+x_{1} \cdot x_{1}+\underset{\uparrow}{x_{2}}+x_{1} \cdot x_{3} \cdot x_{4}+x_{5} \cdot x_{\uparrow}
$$

The first, second, third and fourth regressors

The corresponding variable indexes are given as:

$I_{1}^{\gamma}(1)=1$ and $I_{r(1)}^{\gamma}(1)=1$ with $r(1)=2$;

$I_{r(2)}^{\gamma}(2)=2$ with $r(2)=1$;

$I_{1}^{\gamma}(3)=1, I_{2}^{\gamma}(3)=3$ and $I_{r(3)}^{\gamma}(3)=4$ with $r(3)=3$;

$I_{1}^{\gamma}(4)=5$ and $I_{r(4)}^{\gamma}(4)=7$ with $r(4)=2$.

Hence, the variable index vector can be written as:

$$
\bar{I}_{j}^{\gamma}(t)=[(1,1),(1,1),(2,2),(1,3),(3,3),(4,3),(5,4),(7,4)] \text {. }
$$

\section{B. Fuzzy coefficient determination}

Based on the variable index vector, $\bar{I}_{j}^{\gamma}(t)$ in (13), the fuzzy regression model can be formulated as:

$$
\hat{y}^{\gamma}=b_{0}^{\gamma}+\sum_{i=1}^{n_{j}^{\gamma}(t)} b_{i}^{\gamma} \cdot \prod_{q=1}^{r(i)} x_{I_{q}^{\gamma}(i)}
$$

where $b_{i}^{\gamma}$ with $\gamma=\left\{c^{\prime}, l^{\prime},{ }^{\prime} r^{\prime}\right\}$ are the fuzzy coefficients with respect to $\bar{I}_{j}^{\gamma}(t)$. The three sets of $b_{i}^{\gamma}$ with $\gamma=\left\{c^{\prime},{ }^{\prime} l{ }^{\prime},{ }^{\prime} r^{\prime}\right\}$ are grouped by three fuzzy coefficient vectors as:

$$
\bar{b}_{j}^{\gamma}(t)=\left[b_{0}^{\gamma}, b_{1}^{\gamma}, \ldots, b_{n_{j}^{\gamma}(t)}^{\gamma}\right] \text { with } \gamma=\left\{{ }^{\prime} c^{\prime}, l^{\prime},{ }^{\prime} r^{\prime}\right\} \text {. }
$$

Prior to determining $\bar{b}_{j}^{\gamma}(t)$, a data set, namely $\mathrm{X}_{j}^{\gamma}(t)$, is generated based on $\bar{I}_{j}^{\gamma}(t) . \mathrm{X}_{j}^{\gamma}(t)$ is given as:

$$
\begin{aligned}
\mathrm{X}_{j}^{\gamma}(t) & =\left\{\tilde{y}_{o}(k), p_{0}^{\gamma}(k), p_{1}^{\gamma}(k), \ldots, p_{n_{j}^{\gamma}(t)}^{\gamma}(k) \mid,\right. \\
k & \left.=1,2, \ldots, N_{D}\right\}
\end{aligned}
$$

where $p_{0}^{\gamma}(k)=1$ and $p_{i}^{\gamma}(k)=\prod_{q=1}^{r(i)} x_{I_{q}^{\gamma}(i)}(k)$. All $b_{i}^{\gamma} \in \bar{b}_{j}^{\gamma}(t)$ with $i=1,2, \ldots, n_{j}^{\gamma}(t)$ and $\gamma=\left\{{ }^{\prime} c^{\prime}, l^{\prime} l^{\prime}, r^{\prime}\right\}$ are determined based on the following two approaches, which are either for crisp-output-data or fuzzy-output-data.

\section{1) Crisp-output-data}

For crisp output data (i.e. all $y_{o}^{l}(k)=y_{o}^{r}(k)=0$ ), all $b_{i}^{\gamma}$ are determined by solving the linear programming problem (17a) to $(17 d)$ :

$$
\min J=\sum_{i=0}^{n_{j}^{l}(t)} \sum_{k=1}^{N_{D}} b_{i}^{l} \cdot p_{i}^{l}(k)+\sum_{i=0}^{n_{j}^{r}(t)} \sum_{k=1}^{N_{D}} b_{i}^{r} \cdot p_{i}^{r}(k),
$$

subject to:

$$
\begin{aligned}
& \sum_{i=0}^{n_{j}^{c}(t)} b_{i}^{c} \cdot p_{i}^{c}(k)+(1-h) \cdot \sum_{i=0}^{n_{j}^{l}(t)} b_{i}^{l} \cdot p_{i}^{l}(k) \geq y_{o}^{c}(k), \\
& \sum_{i=0}^{n_{j}^{c}(t)} b_{i}^{c} \cdot p_{i}^{c}(k)-(1-h) \cdot \sum_{i=0}^{n_{j}^{r}(t)} b_{i}^{r} \cdot p_{i}^{r}(k) \leq y_{o}^{c}(k), \\
& \sum_{i=0}^{n_{j}^{l}(t)} b_{i}^{l} \cdot p_{i}^{l}(k) \geq 0, \sum_{i=0}^{n_{j}^{r}(t)} b_{i}^{r} \cdot p_{i}^{r}(k) \geq 0 \text { for all } k=0,1, \ldots, N_{D}
\end{aligned}
$$

Solving $J$ in (17a) attempts to minimize the overall uncertainty estimated by the model. Hence, unnecessary uncertainty is less likely to be estimated. $h$ in (17b) and (17c) is used to adjust the degree to which the model fits the samples, where $h$ is between 0 and 1 . When the constraints $(17 b)$ to $(17 d)$ are satisfactory, the model can cover all the samples with the memberships higher than $h$. The linear programming algorithm is used to solve (17a) to (17d) and determine $b_{i}^{\gamma}$ with $i=1,2, \ldots, n_{j}^{\gamma}(t)$ and $\gamma=c, l$ or $r$. Hence, $\bar{b}_{j}^{\gamma}(t)$ in (15) is determined.

\section{2) Fuzzy-output-data}

For fuzzy output data (i.e. some $y_{o}^{l}(k), y_{o}^{r}(k)>0$ ), all $b_{i}^{\gamma}$ are determined by minimizing the overall residual error, $E$, given in (18).

$$
E=\sum_{k=1}^{N_{D}}\left(\left(\hat{y}^{c}(k), \hat{y}^{l}(k), \hat{y}^{r}(k)\right)-\left(y_{o}^{c}(k), y_{o}^{l}(k), y_{o}^{r}(k)\right)\right)^{2}
$$

where $\hat{y}^{\gamma}(k)$, with $\gamma=\left\{c^{\prime},{ }^{\prime} l{ }^{\prime}, r^{\prime}\right\}$, is the estimate with respect to the $k$-th sample in $D_{j}^{\gamma}(t) . E$ indicates the overall fuzzy distance between the estimates and the samples. (18) can be rewritten as

$$
\begin{aligned}
E= & \left(\sum_{k=1}^{N_{D}}\left(b_{0}^{c}+\sum_{i=1}^{n_{j}^{c}(t)} b_{i}^{c} \cdot p_{i}^{c}(k)\right)-y_{o}^{c}(k), \sum_{k=1}^{N_{D}}\left(b_{0}^{l}+\sum_{i=1}^{n_{j}^{l}(t)} b_{i}^{l} \cdot p_{i}^{l}(k)\right)\right. \\
& \left.-y_{o}^{l}(k), \sum_{k=1}^{N_{D}}\left(b_{0}^{r}+\sum_{i=1}^{n_{j}^{r}(t)} b_{i}^{r} \cdot p_{i}^{r}(k)\right)-y_{o}^{r}(k)\right)
\end{aligned}
$$


After setting the derivatives of (19) with respect to every $b_{i}^{\gamma}$ to zero, three sets of equations with $\gamma=\left\{{ }^{\prime} c^{\prime},{ }^{\prime} l{ }^{\prime},{ }^{\prime} r^{\prime}\right\}$ can be developed [3]. The ordinary least square regression (Chapter 7 of [28]), can be used to solve the three sets of equations. $b_{i}^{c}$ with $i=1,2, \ldots, n_{j}^{\gamma}(t)$ can be determined based on $y_{o}^{c}(k) . b_{i}^{l}$ and $b_{i}^{r}$ can be determined based on $y_{o}^{l}(k)$ and $y_{o}^{r}(k)$, respectively. Hence, all $b_{i}^{\gamma} \in \bar{b}_{j}^{\gamma}(t)$ in (15) can be determined.

\section{Fitness functions}

When $\theta_{j}^{\gamma}(t)$ and $\bar{b}_{j}^{\gamma}(t)$ are given, the fitness of the $j$-th individual is determined by either of the two fitness functions, which are either developed for crisp output data or fuzzy output data. For crisp output data, the mean fuzzy credibility (MFC) [37] is used. For fuzzy output data, the fuzzy number distance (FDIS) [3] is used.

1) Mean fuzzy credibility (MFC)

The MFC is determined based on (20),

$$
\mathrm{MFC}=\sum_{k=1}^{N_{D}} \frac{\mu_{\hat{y}}\left(y_{o}^{c}(k)\right)}{\left|\hat{y}^{r}(k)+\hat{y}^{l}(k)\right|},
$$

where the denominator indicates the overall uncertainty estimated by the model (14); the numerator indicates the fuzzy membership of $y_{o}^{c}(k)$, to the model (14). The fuzzy membership is determined by (1). When MFC is high, the model generates high memberships with respect to all $y_{o}^{c}(k)$ and generates small uncertainty estimates.

2) Fuzzy number distance (FDIS)

For fuzzy output data, the fitness function (21) evaluates the FDIS between the collected samples, $\tilde{y}^{o}(k)=\left(y_{o}^{c}(k), y_{o}^{l}(k), y_{o}^{r}(k)\right)$, and the estimates, $\hat{\tilde{y}}(k)=\left(\hat{y}^{c}(k), \hat{y}^{l}(k), \hat{y}^{r}(k)\right)$, generated by the model (14).

$$
\begin{aligned}
\mathrm{FDIS}= & \sum_{k=1}^{N_{D}}\left\{\left(\hat{y}^{c}(k)-y_{o}^{c}(k)\right)^{2}+\frac{1}{3}\left(\hat{y}^{c}(k)-y_{o}^{c}(k)\right) .\right. \\
& {\left[\left(\hat{y}^{r}(k)-y_{o}^{r}(k)\right)-\left(\hat{y}^{l}(k)-y_{o}^{l}(k)\right)\right]+} \\
& \left.\frac{1}{12}\left[\left(\hat{y}^{r}(k)-y_{o}^{r}(k)\right)^{2}+\left(\hat{y}^{l}(k)-y_{o}^{l}(k)\right)^{2}\right]\right\}
\end{aligned}
$$

(21) is elaborated from (18). The details can be referred to [3].

\section{Parent selection and termination condition}

In $\Omega^{\gamma}(t)$ with $\gamma \in\left\{{ }^{\prime} c^{\prime}, l^{\prime},{ }^{\prime}, r^{\prime}\right\}$, individuals with good fitness are selected as offspring for the following populations $\Omega^{\gamma}(t+1)$. The roulette-wheel approach [30], is used to select the offspring. $\Omega^{\gamma}(t+1)$ evolves iteratively until the generation number, $t$, reaches the termination generation, $G_{T}$.

\section{E. Evolutionary operations}

In NON-SC-FR, the crossover produces two new individuals by inheriting the polynomial structures of two randomly selected individuals. It randomly selects an arithmetical operation in each individual and then it exchanges the sub-polynomial structures at the selected operations. For example, we considered the following individuals, $\theta_{1}$ and $\theta_{2}$, of which the selected arithmetical operations are arrowed:

$$
\begin{aligned}
& \theta_{1}=1+x_{1} \cdot x_{3}+x_{4} \underset{\uparrow}{+} x_{2} \cdot x_{2} \cdot x_{2}+x_{2} \cdot x_{3} \cdot x_{4} \\
& \theta_{2}=1+x_{3}+x_{1}+x_{2} \cdot x_{2}
\end{aligned}
$$

The crossover generates two new individuals as:

$$
\begin{aligned}
& \theta_{1}^{\prime}=1+x_{1} \cdot x_{3}+x_{4}+x_{3}+x_{1}+x_{2} \cdot x_{2} \\
& \theta_{2}^{\prime}=1+x_{2} \cdot x_{2} \cdot x_{2}+x_{2} \cdot x_{3} \cdot x_{4}
\end{aligned}
$$

As different substructures are generated in $\theta_{1}^{\prime}$ and $\theta_{2}^{\prime}$, population diversification can be introduced. Mutation is performed by randomly selecting a character in the individual. The selected character is mutated to a new character. For example, the character of the following individual, $\theta_{3}$, is selected and is arrowed:

$$
\theta_{3}=1+x_{1}+x_{2}+\underset{\uparrow}{x_{3}} \cdot x_{4}
$$

The new character, $x_{1}$, is introduced by the mutation:

$$
\theta_{3}^{\prime}=1+x_{1}+x_{2}+\underset{\uparrow}{x_{1}} \cdot x_{4}
$$

\section{EVALUATIONS OF ALGORITHMIC EFFECTIVENESS}

Every day, huge amounts of digital images are captured by digital cameras, smartphones or tablets. The captured images need to be a faithful representation of the scenes people wish to capture. IQA is essential to evaluate the aesthetic quality of images before performing many image processing applications [12]. Therefore, IQA models are necessary to predict aesthetic qualities of captured images [13]. Our previous research developed the fuzzy regression approaches to generate IQA models [5,6]. The approaches attempt to overcome the limitation of the commonly used statistic regression and classical fuzzy regression, which cannot properly estimate uncertainty of IQA [5,6], but the approaches can only generates the linear fuzzy centers which are linearly correlated to the independent variables. Here the proposed NON-SC-FR is used to further enhance the robustness of IQA models, where the centers of the fuzzy estimates are generated by the genetic programming and they can be nonlinearly correlated to the independent variables. Also, the effectiveness of NON-SC-FR can be evaluated by developing the IQA models.

Two IQA databases, TID database [50] and VCL database [69], have been used to develop the IQA. These two databases are used because they consist of different data types, namely crisp-outputdata and fuzzy-output-data. TID database contains the mean opinion score (MOS) for each image. Hence the samples are in crisp-output-data as the MOS is a real number. The effectiveness of the proposed NON-SC-FR in dealing with crisp-output-data can be evaluated through TID database. VCL database contains a full set of individual opinion scores, which are evaluated by each participant to each image. Hence this set of full samples can be transformed into fuzzy-output-data. The effectiveness of NON-SCFR in dealing with fuzzy-output-data can be evaluated through the VCL database.

The proposed NON-SC-FR was implemented based on the routines of GP Matlab package [40] which is available for the public. The following GP parameters [40], which are effective for developing polynomial models, were used: population size $=50$; maximum number of generations $=500$; crossover rate $=0.5$; mutation rate $=0.5$.

As NON-SC-FR is a stochastic method, different results are generated from different runs. To evaluate its overall performance, 
30 runs are performed on NON-SC-FR. The result for the median of the 30 runs is used as the comparison. The proposed NON-SCFR is compared with the recently-developed fuzzy regression methods namely LW-FR [39], HW-FR [20], GP-FR [4] and U-FR [61], where they are particularly developed to address the nonlinear or varying uncertainty of training samples. LW-FR and HW-FR are developed for both fuzzy and crisp output-data; GP-FR is developed for crisp-output-data; U-FR is developed for fuzzyoutput-data.

LW-FR [39] is developed, in order to overcome the deficiency of previous fuzzy regressions which assume uncertainty for dependent variables increase with increasing magnitudes of independent variables. The LW-FR fits the uncertainty of dependent variable which can be increased or decreased with increasing magnitudes of independent variables.

HW-FR [20] is developed based on a polynomial with quadratic terms, in order to fit sample nonlinearity. Experimental results showed that HW-FR is more effective on short term load forecasting than the state-of-art fuzzy regression.

GP-FR [4] uses GP to develop polynomial models with high order and interaction terms, in order to model nonlinearity of manufacturing systems. The effectiveness was demonstrated by solving a manufacturing design problem. As GP-FR is a heuristic method, 30 runs are performed on GP-FR and the median results are used to compare with those of the proposed NON-SC-FR.

U-FR [61] is developed based on high order polynomials to represent the central, left and right spreads of the fuzzy regression models. U-FR takes into account the possible interactions between uncertainties and magnitudes of dependent variables. The effectiveness of U-FR was demonstrated by modelling software reliability.

\section{A. Crisp-output-data}

\section{1) Description for TID database}

In the TID database, 25 high quality images were used to generate 2125 distorted images which were contaminated by 17 types of

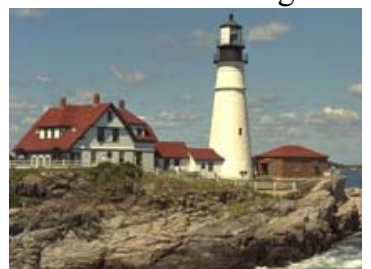

Fig. 4a JPEG in level 1

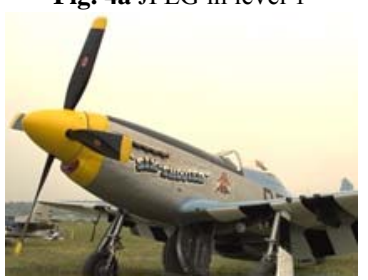

Fig. 5a JPEG2000 in level 1

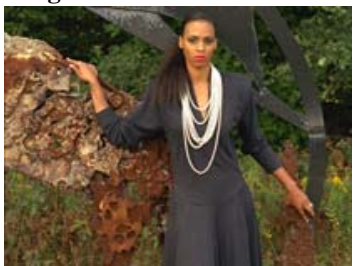

Fig. 6a Impulse noise in level 1

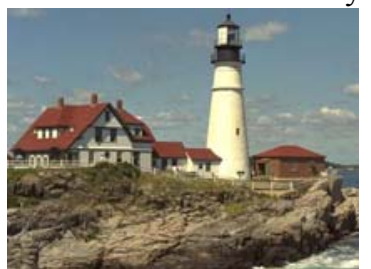

Fig. 4b JPEG in level 2
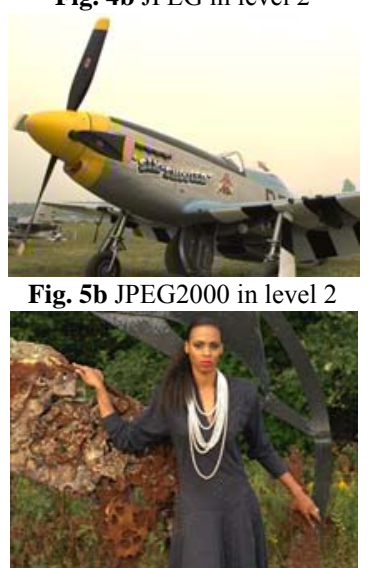

Fig. 6b Impulse noise in level 2

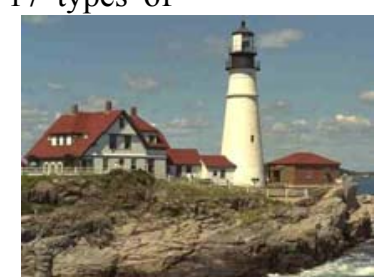

Fig. 4c JPEG in level 3

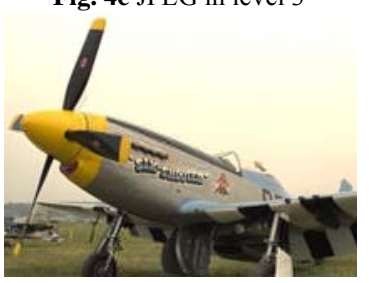

Fig. 5c JPEG2000 in level 3

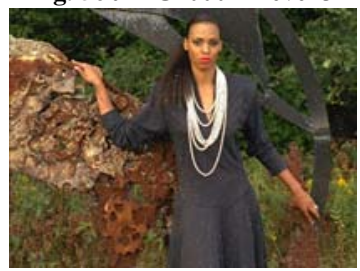

Fig. 6c Impulse noise in level 3

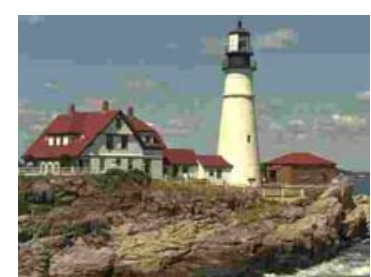

Fig. 4d JPEG in level 4

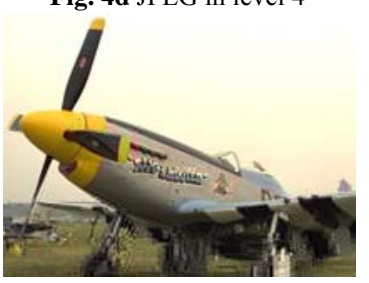

Fig. 5d JPEG2000 in level 4

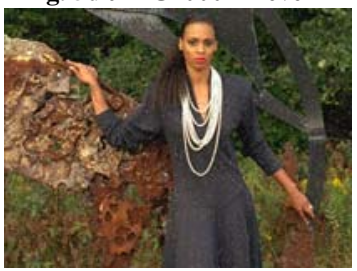

Fig. 6d Impulse noise in level 4

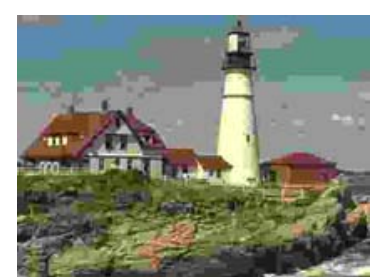

Fig. 4e JPEG in level 5

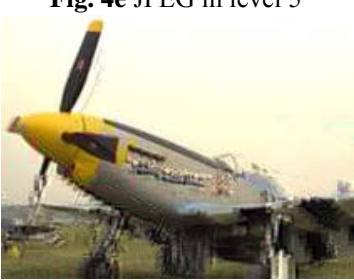

Fig. 5e JPEG2000 in level 5

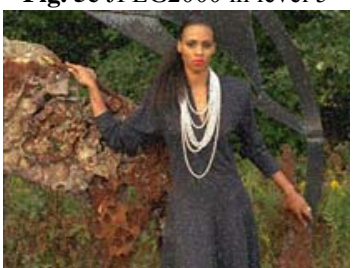

Fig. 6e Impulse noise in level 5 
Table 2 Distortion types of TID database and the five sets of distorted types, JPEG, Noise, Actual, Exotic and Full (the table is originally from [50])

\begin{tabular}{|c|c|c|c|c|c|}
\hline & \multicolumn{5}{|c|}{ Image sets } \\
\hline Distortion types & JPEG & Noise & Actual & Exotic & Full \\
\hline Additive Gaussian noise & Excluded & Included & Included & Excluded & Included \\
\hline $\begin{array}{l}\text { Different additive noise in colour } \\
\text { components }\end{array}$ & Excluded & Excluded & Excluded & Excluded & Included \\
\hline Spatially correlated noise & Excluded & Included & Included & Excluded & Included \\
\hline Masked noise & Excluded & Excluded & Excluded & Excluded & Included \\
\hline High frequency noise & Excluded & Included & Excluded & Excluded & Included \\
\hline Impulse noise & Excluded & Included & Included & Excluded & Included \\
\hline Quantization noise & Excluded & Excluded & Included & Excluded & Included \\
\hline Gaussian blur & Excluded & Included & Included & Excluded & Included \\
\hline Image denoising & Excluded & Included & Included & Excluded & Included \\
\hline JPEG compression & Included & Excluded & Included & Excluded & Included \\
\hline JPEG2000 compression & Included & Excluded & Included & Excluded & Included \\
\hline JPEG transmission errors & Excluded & Excluded & Excluded & Excluded & Included \\
\hline JPEG2000 transmission errors & Excluded & Excluded & Excluded & Excluded & Included \\
\hline Non eccentricity pattern noise & Excluded & Excluded & Excluded & Included & Included \\
\hline Local blockwise distortions & Excluded & Excluded & Excluded & Included & Included \\
\hline Mean shift & Excluded & Excluded & Excluded & Included & Included \\
\hline Contrast change & Excluded & Excluded & Excluded & Included & Included \\
\hline Most significant objective features & PSNRHVSM $x_{11}$ & PSNRHVS $x_{8}$ & PSNRHVSM $x_{11}$ & $\operatorname{MSSIM} x_{2}$ & $\operatorname{MSSIM} x_{2}$ \\
\hline Numbers of MOS samples & 250 & 750 & 1000 & 500 & 2125 \\
\hline
\end{tabular}

Figures $7 \mathrm{a}$ to $7 \mathrm{~d}$ illustrate the model characteristics for the image set, Exotic. They show that the spreads of the proposed NON-SCFR better fit the samples than the spreads of the existing methods. As the spreads illustrate the estimated uncertainty, unnecessary amount of uncertainty is unlikely to be estimated by the NON-SCFR. For HW-FR, which can only develop infeasible solutions, Figure $7 \mathrm{~b}$ shows that not all the samples can be covered by the spreads. Figures $8 \mathrm{a}$ to $8 \mathrm{~d}$ show the similar results for the image set, Full. The proposed NON-SC-FR achieves better sample fitting than the existing methods do. It shows that unnecessary amount of uncertainty is less likely to be estimated by NON-SC-FR. Hence it further validates that NON-SC-FR outperforms the three tested methods.

Table 4 shows the fuzzy criteria, MFC, for the model which engages only the most significant feature of each of the five image sets, JPEG, Noise, Actual, Exotic and Full, where the MFC is formulated in (21). These results indicate the effectiveness and the performance of the proposed NON-SC-FR in generating the fuzzy regression models, compared with other tested methods. Also the ranks of the four tested methods are given with respect to the MFC which is the-larger-the-better. They show that the proposed NONSC-FR obtains higher MFCs and higher ranks compared with the other tested methods. The proposed NON-SC-FR can generate models which have better fitting capability to the MOS data than the other tested methods.

Table 3 IQA models for TID datasets

\begin{tabular}{|c|c|c|}
\hline \multirow{4}{*}{ 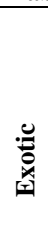 } & LW-FR & $\tilde{y}=(0.26,4.32,4.34)+\left(4.93,1.04 \times 10^{-9}, 1.04 \times 10^{-9}\right) x_{2}$ \\
\hline & HW-FR & $\tilde{y}=(1.05,0.90)+(0.66,0.90) x_{2}+(4.00,0.90) x_{2}^{2}$ \\
\hline & GP-FR & $\tilde{y}=(1.58,1.62)+(4.10,1.62) \cdot x_{2}^{4}$ \\
\hline & $\begin{array}{l}\text { NON-SC- } \\
\text { FR }\end{array}$ & $\tilde{y}=\left(2.29+2.496 \cdot x_{2}^{4},-2.68+6.06 \cdot x_{2}, 0.91+0.33 \cdot x_{2}^{2}\right)$ \\
\hline \multirow{4}{*}{$\bar{\Xi}$} & LW-FR & $\tilde{y}=(-0.17,5.18,5.20)+\left(5.19,2.30 \times 10^{-13}, 2.4 \times 10^{-13}\right) x_{2}$ \\
\hline & HW-FR & $\tilde{y}=(0.80,1.07)+(0.29,1.07) \cdot x_{2}+(4.19,1.06) \cdot x_{2}^{2}$ \\
\hline & GP-FR & $\tilde{y}=(-1.00,1.88)+(6.07,1.88) \cdot x_{2}^{2}$ \\
\hline & $\begin{array}{l}\text { NON-SC- } \\
\text { FR }\end{array}$ & $\begin{aligned} \tilde{y}= & \left(13.79-39.66 \cdot x_{2}+30.46 \cdot x_{2}^{2},-12.94+39.06 \cdot x_{2}\right. \\
& \left.-23.94 \cdot x_{2}^{2},-2.37+5.12 \cdot x_{2}\right)\end{aligned}$ \\
\hline
\end{tabular}

Despite the MFC, two other fuzzy criteria, namely average fuzzy spread (AFS) [57] and index of confidence (IC) [63], are used to further validate the effectiveness and the performance of the fuzzy regressions, and they are described as following:

AFS [57] given in (22) indicates the overall uncertainty predicted by the model. Unnecessary uncertainty is unlikely to be predicted by the model, when AFS is small.

$$
\mathrm{AFS}=\sum_{k=1}^{N_{D}} \Delta \hat{\tilde{y}}(k)=\sum_{k=1}^{N_{D}}\left|\hat{y}^{r}(k)-\hat{y}^{l}(k)\right|
$$

IC [63] given in (23) evaluates the degree of variation of all collected samples $\tilde{y}_{o}(k)=\left(y_{o}^{c}(k), y_{o}^{l}(k), y_{o}^{r}(k)\right)$ to all fuzzy estimates $\hat{\tilde{y}}(k)=\left(\hat{y}^{c}(k), \hat{y}^{l}(k), \hat{y}^{r}(k)\right)$ with $k=$ $1,2, \ldots, N_{D}$ which are the model outputs. The IC is similar to the $R$-square in statistical regression, which indicates the capability of fitting the collected samples. When the IC is large, the fitting capability of the fuzzy regression model is better.

$$
\mathrm{IC}=\frac{\sum_{k=1}^{N_{D}}\left(\hat{y}^{c}(k)-\hat{y}^{l}(k)\right)^{2}+\sum_{k=1}^{N_{D}}\left(\hat{y}^{c}(k)-\hat{y}^{r}(k)\right)^{2}}{\sum_{k=1}^{N_{D}}\left(y_{o}^{c}(k)-\hat{y}^{l}(k)\right)^{2}+\sum_{k=1}^{N_{D}}\left(y_{o}^{c}(k)-\hat{y}^{r}(k)\right)^{2}},
$$

Tables 5 and 6 show the results for AFS and IC respectively of which the models are engaged with the most significant feature. Table 5 shows that the proposed NON-SC-FR generates smaller AFSs than the other tested methods, LW-FR, HW-FR and GP-FR. The ranks of the proposed NON-SC-FR are the highest. Hence, the NON-SC-FR is capable to develop models, which are unlikely to estimate unnecessary amount of uncertainty than the other methods. Table 6 shows that NON-SC-FR obtains better ICs than those obtained by the other tested methods. NON-SC-FR can generate models with better fitting capability to the MOS samples. Therefore, the proposed NON-SC-FR is better than the other tested methods when these three criteria, MFC, AFS and IC, are taken into account. 


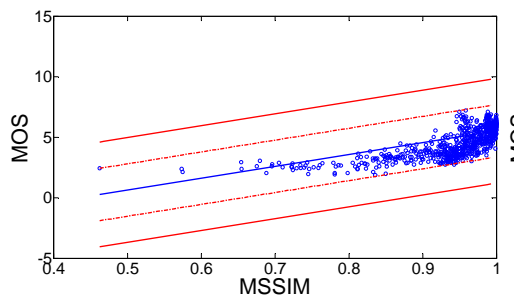

Figure 7a LW-FR for exotic

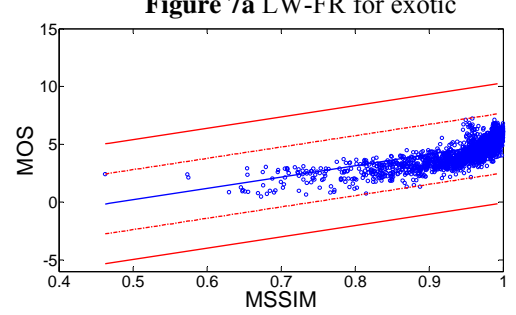

Figure 8a LW-FR for full

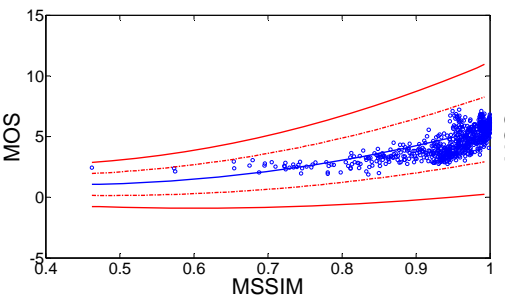

Figure 7b HW-FR for exotic

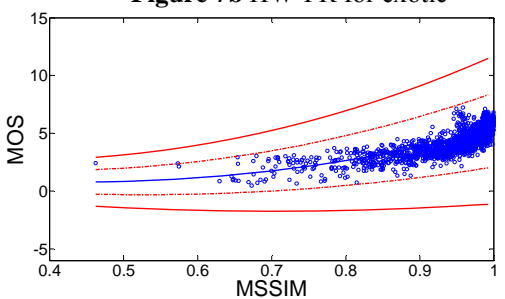

Figure 8b HW-FR for full

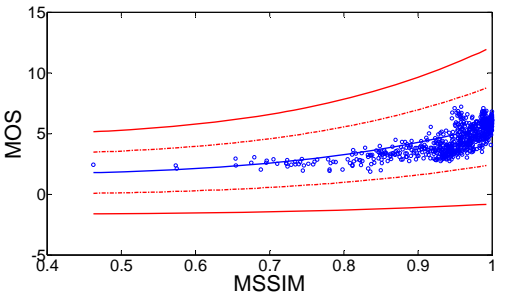

Figure 7c GP-FR for exotic

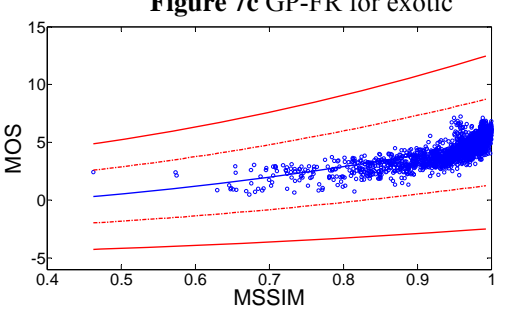

Figure 8c GP-FR for full

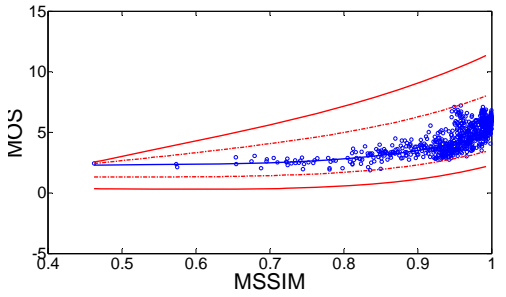

Figure 7d NON-SC-FR for exotic

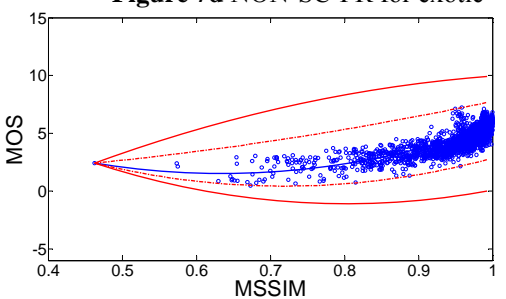

Figure 8d NON-SC-FR for full

Table 4 MFC for the most significant IQA metric

\begin{tabular}{|c|c|c|c|c|}
\hline & LW- & HW- & GP- & NON- \\
FR & FR & FR & SC-FR \\
\hline JPEG & 0.15 & 0.33 & 0.28 & 0.37 \\
Noise & 0.11 & 0.19 & 0.17 & 0.25 \\
Exotic & 0.09 & 0.13 & 0.11 & 0.16 \\
Actual & 0.10 & 0.17 & 0.14 & 0.20 \\
Full & 0.08 & 0.13 & 0.10 & 0.16 \\
Ave.MFC & $\mathbf{0 . 1 1}$ & $\mathbf{0 . 1 9}$ & $\mathbf{0 . 1 6}$ & $\mathbf{0 . 2 3}$ \\
Rank & $\mathbf{4}$ & $\mathbf{2}$ & $\mathbf{3}$ & $\mathbf{1}$ \\
\hline
\end{tabular}

MFC is the-larger-the-better

Table 7 MFC for the MOS to all IQA metrics

\begin{tabular}{|c|c|c|c|c|}
\hline & $\begin{array}{c}\text { LW- } \\
\text { FR }\end{array}$ & $\begin{array}{c}\text { HW- } \\
\text { FR }\end{array}$ & $\begin{array}{c}\text { GP- } \\
\text { FR }\end{array}$ & $\begin{array}{c}\text { NON- } \\
\text { SC-FR }\end{array}$ \\
\hline JPEG & 0.29 & 1.32 & 0.19 & 4.28 \\
Noise & 0.20 & 0.75 & 0.21 & 1.07 \\
Exotic & 0.14 & 0.93 & 0.15 & 3.68 \\
Actual & 0.16 & 0.80 & 0.20 & 2.24 \\
Full & 0.14 & 0.39 & 0.16 & 0.93 \\
Ave.MFC & $\mathbf{0 . 1 9}$ & $\mathbf{0 . 8 4}$ & $\mathbf{0 . 1 8}$ & $\mathbf{2 . 4 4}$ \\
Rank & $\mathbf{3}$ & $\mathbf{2}$ & $\mathbf{4}$ & $\mathbf{1}$ \\
\hline
\end{tabular}

MFC is the-larger-the-better

Tables 7, 8 and 9 show the results for MFC, AFS and IC of which the models are engaged with all the seventeen features, $x_{1}$ to $x_{17}$. Based on these results, we can evaluate the effectiveness of the algorithm when more number of independent variables is involved. The tables show that the NON-SC-FR can generate better AFS, IC and MFC than those generated by LW-FR, HW-FR and GP-FR. Therefore, the proposed NON-SC-FR is capable of generating better models when more independent variables are involved. Better results obtained by the proposed NON-SC-FR can be explained by the mechanism of the NON-SC-FR, which can generate more flexible polynomials to represent the right and left spreads and also the center. The generated models can be better fitted to the MOS samples and they are developed with smaller fuzzy intervals, which are less likely to estimate unnecessary uncertainty. Therefore, the proposed NON-SC-FR is able to obtain better IC, AFS and MFC than those obtained by LW-FR, HW-FR and GP-FR.

\section{B. Fuzzy-output-data}

1) Description for VCL database

VCL database [69] contains 552 distorted images. These distorted images are generated based on the 23 reference images with two distortion sets. The first set, namely Compression, was involved
Table 6 IC for the most significant IQA metric

\begin{tabular}{|c|c|c|c|c|}
\hline & $\begin{array}{c}\text { LW- } \\
\text { FR }\end{array}$ & $\begin{array}{c}\text { HW- } \\
\text { FR }\end{array}$ & $\begin{array}{c}\text { GP- } \\
\text { FR }\end{array}$ & $\begin{array}{c}\text { NON- } \\
\text { SC-FR }\end{array}$ \\
\hline JPEG & 0.24 & 0.79 & 0.54 & 0.82 \\
Noise & 0.25 & 0.67 & 0.56 & 0.86 \\
Exotic & 0.24 & 0.80 & 0.59 & 0.89 \\
Actual & 0.24 & 0.73 & 0.55 & 0.89 \\
Full & 0.24 & 0.72 & 0.63 & 0.83 \\
Ave.IC & $\mathbf{0 . 2 4}$ & $\mathbf{0 . 7 4}$ & $\mathbf{0 . 5 7}$ & $\mathbf{0 . 9 4}$ \\
Rank & $\mathbf{4}$ & $\mathbf{2}$ & $\mathbf{3}$ & $\mathbf{1}$ \\
\hline
\end{tabular}

IC is the-larger-the-better

Table 9 IC for the MOS to all IQA metrics

\begin{tabular}{|c|c|c|c|c|}
\hline & $\begin{array}{c}\text { LW- } \\
\text { FR }\end{array}$ & $\begin{array}{c}\text { HW- } \\
\text { FR }\end{array}$ & $\begin{array}{c}\text { GP- } \\
\text { FR }\end{array}$ & $\begin{array}{c}\text { NON- } \\
\text { SC-FR }\end{array}$ \\
\hline JPEG & 0.23 & 0.81 & 0.78 & 0.89 \\
Noise & 0.24 & 0.80 & 0.75 & 0.87 \\
Exotic & 0.24 & 0.79 & 0.74 & 0.83 \\
Actual & 0.24 & 0.81 & 0.77 & 0.88 \\
Full & 0.24 & 0.82 & 0.81 & 0.87 \\
Ave.IC & $\mathbf{0 . 2 4}$ & $\mathbf{0 . 8 1}$ & $\mathbf{0 . 7 7}$ & $\mathbf{0 . 8 7}$ \\
Rank & $\mathbf{4}$ & $\mathbf{2}$ & $\mathbf{3}$ & $\mathbf{1}$ \\
\hline
\end{tabular}

IC is the-larger-the-better

with JPEG and JPEG2000 compressions. The second set, namely Blur, was involved with Additive White Gaussian Noise and Gaussian Blur. Each distorted image was contaminated by certain distortion of which six levels of distortion were contaminated. The image qualities for the distorted images were scored by 118 persons of which their ages were between 20 and 30 years. 14 to 36 scores were collected for each image and each image was scored with an average of 20 times.

VCL database contains each individual opinion score obtained from each participant while the TID database only contains the averaged opinion score for each image. Hence VCL database can be used to generate fuzzy MOS in fuzzy number for each image. The fuzzy MOS for the $k$-th distorted image is defined as the fuzzy number, $\tilde{y}_{o}(k)=\left(y_{o}^{c}(k), y_{o}^{l}(k), y_{o}^{r}(k)\right)$, as:

$$
\begin{aligned}
& y_{o}^{c}(k)=\frac{1}{N_{s}(k)} \sum_{j=1}^{N_{s}(k)} y_{j}(k) ; \\
& y_{o}^{l}(k)=y_{o}^{c}(k)-\min _{j=1,2, ., N_{s}(k)}\left(y_{j}(k)\right) ; \\
& \tilde{y}_{o}^{r}(k)=\max _{j=1,2, \ldots, N_{s}(k)}\left(y_{j}(k)\right)-\tilde{y}_{o}^{c}(k) ;
\end{aligned}
$$

$y_{j}(k)$ is the opinion score given by the $j$-th participant for the $k$-th distorted image; $N_{s}(k)$ is the number of participants involved on 
scoring the $k$-th distorted image. Using these fuzzy MOS samples, we can evaluate the effectiveness of NON-SC-FR in dealing with fuzzy-output-data.

\section{2) Experimental results}

Table 10 illustrates the characteristics of IQA models developed based on LW-FR, HW-FR, U-FR and the proposed NON-SC-FR, where the models are developed for Compression and Blur. The most significant features, PSNRHVSM $x_{11}$ and MSSIM $x_{2}$ respectively are correlated to the Compression and the Blur models respectively. In Figures $9 \mathrm{a}$ to $9 \mathrm{~d}$, the characteristics of the Compression models are illustrated. The proposed NON-SC-FR and U-FR better fit the fuzzy MOS samples than the other two existing methods, LW-FR and HW-FR. Similarly, Figures 10a to 10d show the models for Blur. They also show that the U-FR and NON-SC-FR outperform LW-FR and HW-FR. These figures show that the fitting capabilities of U-FR and the proposed NON-SC-FR are similar. We cannot distinguish the difference regarding the fitting capability for U-FR and NON-SC-FR.

To further distinguish the fitting capability of the models, the FDIS in (21) is used. The first main row of Table 11 shows fitting capabilities of the models. It shows the FDISs obtained by the models for JPEG, Blur and the average of two. It shows that smallest FDIS is obtained by the proposed NON-SC-FR compared with LW-FR, HW-FR and U-FR. The ranks achieved by the NONSC-FR are the highest. The fuzzy MOS estimated by the proposed NON-SC-FR are closest to the collected fuzzy MOS samples. Hence, the fitting capability of the proposed NON-SC-FR is better than that of the other methods. To evaluate the generalization capability of the models, we performed the Leave-one-out-crossvalidation (LOOCV). Towards that end, the fuzzy MOS samples contaminated from 22 reference images are used for training and the samples contaminated from the remaining one reference image are used for validation. The LOOCV results are shown in the

second main row of Table 11. These results confirm that the proposed NON-SC-FR performs consistently better than the other tested methods also on validation sets.

Table 10 IQA models for VCL dataset

\begin{tabular}{|c|c|c|}
\hline \multirow{4}{*}{ 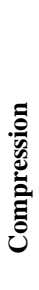 } & LW-FR & $\tilde{y}=\left(2.07 \cdot x_{11}-9.65,0.24 \cdot x_{11}+18.5,0.44 \cdot x_{11}+35.24\right)$ \\
\hline & HW-FR & $\tilde{y}=(-13.0,19.5)+(189,19.4) \cdot x_{11}+(-73,19.5) \cdot x_{11}^{2}$ \\
\hline & U-FR & $\begin{array}{l}\tilde{y}=\left(y^{c}, 8.11+0.19 \cdot y^{c}, 5.84+0.23 \cdot y^{c}\right) \\
\text { where } y^{c}=338-56.71 \cdot x_{11}+3.62 \cdot x_{11}^{2}-0.10 \cdot x_{11}^{3}\end{array}$ \\
\hline & $\begin{array}{l}\text { NON- } \\
\text { SC-FR }\end{array}$ & $\begin{aligned} \tilde{y}= & \left(-13.0+0.1 \cdot x_{11}+0.1 \cdot x_{11}^{2},-5 .+0.89 \cdot x_{11}\right. \\
& \left.-2.8 \times 10^{-6} \cdot x_{11}^{3}, 20.1+0.03 \cdot x_{11}^{2}\right)\end{aligned}$ \\
\hline \multirow{4}{*}{$\stackrel{\Xi}{M}$} & LW-FR & $\tilde{y}=\left(110.4 \cdot x_{2}-48.5,14.7 \cdot x_{2}+13.8,-3.0 \cdot x_{2}+24.7\right)$ \\
\hline & HW-FR & $\tilde{y}=(19.3,13.9)+(-79.7,13.9) \cdot x_{2}+(123.8,13.9) \cdot x_{2}^{2}$ \\
\hline & U-FR & $\begin{aligned} \tilde{y} & =\left(y^{c}, 5.29+0.25 \cdot y^{c}, 6.44+0.23 \cdot y^{c}\right), \text { where } y^{c}=-158 \\
& +10^{3} \cdot\left(1.8 \cdot x_{2}-7.5 \cdot x_{2}^{2}+14.6 \cdot x_{2}^{3}-13.3 \cdot x_{2}^{4}+46.9 \cdot x_{2}^{5}\right)\end{aligned}$ \\
\hline & $\begin{array}{l}\text { NON- } \\
\text { SC-FR }\end{array}$ & $\begin{aligned} \tilde{y}= & \left(14.18+10.49 \cdot x_{2}-93.78 \cdot x_{2}^{2}+36.05 \cdot x_{2}^{3},\right. \\
& \left.12.87+38.50 \cdot x_{2}, 12.97+38.41 \cdot x_{2}\right)\end{aligned}$ \\
\hline
\end{tabular}

Table 11 Fuzzy number distances (FDISs) for fuzzy regressions

\begin{tabular}{|c|c|c|c|c|c|}
\hline & & LW-FR & HW-FR & U-FR & NON-SC-FR \\
\hline \multirow{4}{*}{ 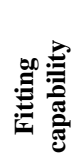 } & JPEG & 8.81 & 12.71 & 6.43 & 4.48 \\
\hline & Blur & 37.92 & 15.60 & 6.10 & 4.22 \\
\hline & Ave. & 23.37 & 14.16 & 6.27 & 4.35 \\
\hline & Rank & 4 & 3 & 2 & 1 \\
\hline \multirow{4}{*}{ ப̊ } & JPEG & 10.29 & 18.61 & 5.93 & 4.99 \\
\hline & Blur & 10.03 & 6.66 & 5.91 & 5.49 \\
\hline & Ave. & 10.16 & 12.64 & 5.92 & 5.24 \\
\hline & Rank & 3 & 4 & 2 & 1 \\
\hline
\end{tabular}

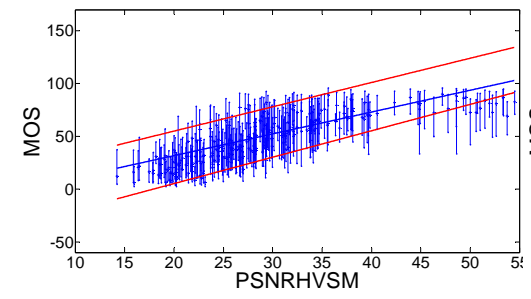

Fig. 9a LW-FR for Compression

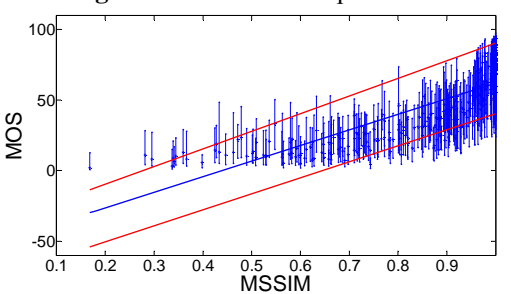

Fig. 10a LW-FR for Blur

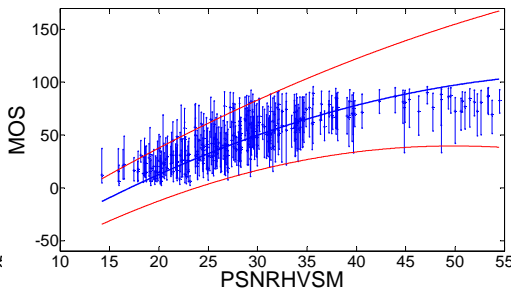

Fig. 9b HW-FR for Compression

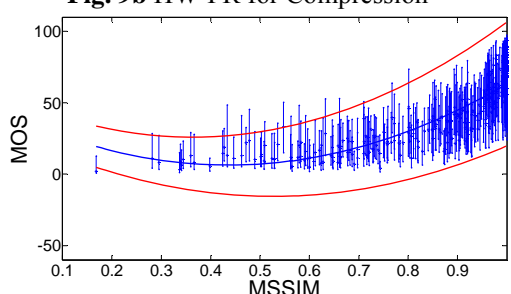

Fig. 10b HW-FR for Blur

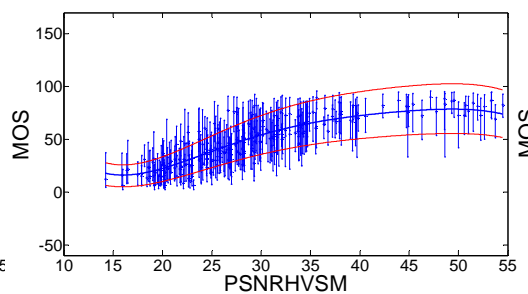

Fig. 9c U-FR for Compression

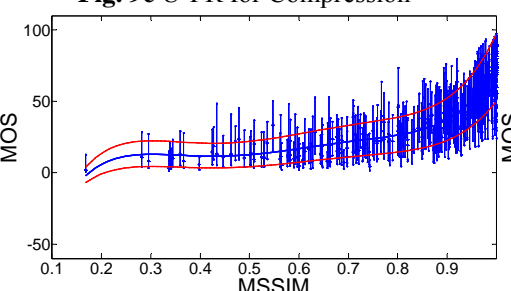

Fig. 10c U-FR for Blur

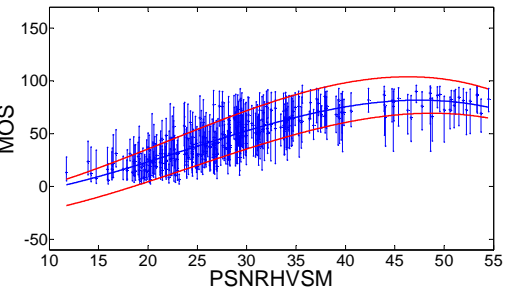

Fig. 9d NON-SC-FR for Compression

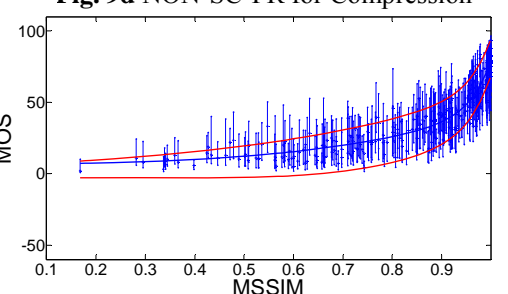

Fig. 10d NON-SC-FR for Blur

\begin{tabular}{|c|c|c|c|c|c|c|c|c|c|}
\hline \multirow{2}{*}{$\begin{array}{l}\text { Fuzzy } \\
\text { regression } \\
\text { methods }\end{array}$} & \multicolumn{4}{|c|}{ Fuzzy criteria } & \multicolumn{5}{|c|}{ Modelling characteristics } \\
\hline & MFC & AFS & IC & FDIS & $\begin{array}{l}\text { Number of } \\
\text { independent } \\
\text { variables }\end{array}$ & $\begin{array}{l}\text { Magnitude } \\
\text { modelling }\end{array}$ & Uncertainty modelling & Data types & $\begin{array}{l}\text { Algorithmic } \\
\text { types }\end{array}$ \\
\hline LW-FR & Rank 3 & Rank 4 & Rank 4 & Rank 3 & More than 1 & Linear & Decreasing or increasing & Fuzzy/crisp & Deterministic \\
\hline HW-FR & Rank 2 & Rank 2 & Rank 2 & Rank 4 & More than 1 & Nonlinear & Only increasing & Fuzzy/crisp & Deterministic \\
\hline GP-FR & Rank 4 & Rank 3 & Rank 3 & Nil & More than 1 & Nonlinear & Only increasing & Crisp & Heuristic \\
\hline U-FR & Nil & Nil & Nil & Rank 2 & Restricted to 1 & Nonlinear & Decreasing or increasing & Fuzzy/crisp & Deterministic \\
\hline $\begin{array}{l}\text { Proposed } \\
\text { NON-SC-FR }\end{array}$ & Rank 1 & Rank 1 & Rank 1 & Rank 1 & More than 1 & Nonlinear & Decreasing or increasing & Fuzzy/crisp & Heuristic \\
\hline
\end{tabular}




\section{Discussion}

Sections IV.A and IV.B show that the proposed NON-SC-FR is better than the four other tested methods, LW-FR, HW-FR, GP-FR and U-FR, when the four fuzzy criteria, MFC, AFS, IC, and FDIS are taken into account. This section attempts to give a tradeoff between the five methods. Here Table 12 summarizes the performance and the characteristics of the proposed NON-SC-FR and the four existing methods.

The left hand main column of Table 12 summarizes the fuzzy criteria achieved by the four tested methods. It gives the ranks provided in Tables 7, 8, 9 and 11. The columns with MFC, AFS and IC present the results achieved for developing the models with respect to all IQA metrics. The column of FDIS presents the results for LOOCV. Table 12 shows that the proposed NON-SC-FR has the highest ranks for these four criteria, MFC, AFS, IC and FDIS. Despite the four criteria, modelling characteristics of the four existing methods and the proposed NON-SC-FR are also given. First, the right hand main column of Table 12 shows that the proposed NON-SC-FR, LW-FR, HW-FR and GP-FR can be used to generate models with more than one independent variable. U-FR can only generate models which are restricted with one independent variable. Hence, this is the limitation of the U-FR. The proposed NON-SC-FR is more flexible than the U-FR.

Then the right hand main column of Table 12 shows that the proposed NON-SC-FR, HW-FR, GP-FR and U-FR can model the nonlinear magnitudes of the aesthetic quality. LW-FR can only model the linear magnitudes. It also shows that the proposed NONSC-FR can model the decreasing or increasing characteristics of the uncertainty when the magnitudes of independent variables are increased. The HW-FR and GP-FR can only model the increasing characteristic of the uncertainty which is the common limitation of the classical fuzzy regression. Also the proposed NON-SC-FR can be used develop models when either fuzzy or crisp data is given. Although the proposed NON-SC-FR is the heuristic method, the aesthetic quality design can be performed off line and they are not necessary to be performed in a real time. Hence the heuristic methods can be used. Also the proposed NON-SC-FR is generally more feasible than the other methods in terms of the other characteristics and it can obtain better results when the four fuzzy criteria are taken into account. This is tradeoff in selecting the proposed method for developing aesthetic quality models.

\section{Conclusion}

The paper proposed a novel fuzzy regression, NON-SC-FR, to estimate the magnitudes and uncertainties caused by aesthetic quality assessments, while the commonly used statistical regression can estimate only the magnitudes. Also the NON-SC-FR overcomes the deficiency of fuzzy regressions which address only one characteristic where the estimated uncertainty can only increase with the increasing magnitudes of objective features. In fact, quality uncertainty can be smaller or unchanged when the feature magnitudes are increased. The proposed NON-SC-FR is incorporated with GP in order to generate the central, and also the right and left spreads, of the model structures, where the central addresses the quality magnitude and the spreads address the quality uncertainty. As GP is effective on fitting nonlinear samples, nonlinearities of quality magnitudes and uncertainties can be addressed effectively by the proposed NON-SC-FR. After the model structures are determined, the fuzzy coefficients are optimized by the regression criteria. It further enhances the fitting capability of model.
The effectiveness and the performance of the proposed NONSC-FR are evaluated by two databases for IQA, where the IQA is necessary for image processing. We consider the IQA problem due to the following reasons: IQA is involved with nonlinear magnitudes and uncertainties; the two IQA databases contain different types of samples (one for crisp-output-data and the other for fuzzy-output-data); the databases consist of significant amounts of samples. Hence different characteristics of human assessments can be taken into account. The experimental results obtained by the proposed NON-SC-FR were compared with four other fuzzy regression methods, which are developed to overcome the aforementioned deficiency of uncertainty estimation. Better results were obtained by the NON-SC-FR when the four criteria, namely confidence index, fuzzy credibility, fuzzy spread and fuzzy number distance are taken into account.

In the future, we will use EEG signals as an objective feature which is correlated to the perceptual quality. As EEG signal is highly imprecise, this imprecision can be represented by the measure tolerances. Based on the measure tolerances, samples in fuzzy numbers can be developed for the independent variables. As the samples on both dependent and independent variables are in fuzzy numbers, we can integrate Khan et al's fuzzy regression method [26] to determine the fuzzy coefficients in Section III.B.2, where Khan et al's approach can be used to develop fuzzy regression models which correlate fuzzy independent variables to fuzzy dependent variables and also it can address the non-triangular fuzzy numbers. Based on Khan et al's approach, the fuzzy coefficients can also be represented in non-triangular fuzzy numbers and the responses of the dependent variables are also in non-triangular fuzzy numbers. Hence, this will be more flexible than using triangular fuzzy numbers, and it will enhance the capability to model the nonlinear characteristics of the uncertainty.

\section{REFERENCES}

[1] M.C. Beardsley, What is an aesthetic quality? Theoria, vol. 39, no. 1-3, pp. 5070, 1973.

[2] J.J. Buckely and T. Feuring, Linear and non-linear fuzzy regression: Evolutionary algorithm solutions, Fuzzy Sets and Systems, vol. 112, no. 3, pp. 381-394, 2000

[3] Y.H. Chang, Hybrid fuzzy least squares regression analysis and its reliability measures, Fuzzy Sets and Systems, vol. 119, pp. 225-246, 2001.

[4] K.Y. Chan, C.K. Kwong and Y.C. Tsim, A genetic programming based fuzzy regression approach to modelling manufacturing processes, International Journal of Production Research, vol. 48, no. 7, pp. 1967-1982, 2010.

[5] K.Y. Chan and U. Engelke, Fuzzy regression for perceptual image quality assessment, Engineering Applications of Artificial Intelligence, vol. 43, pp. 102$110,2015$.

[6] K.Y. Chan and U. Engelke, Varying spread fuzzy regression for affective quality estimation, Engineering IEEE Transactions on Fuzzy Systems, 2016.

[7] H.C. Chen, J.H. Fang, S.M.E. Kortright and D.S. Chen, Novel approaches to the determination of archie parameters II: fuzzy regression analysis, SPE: Advanced Technology Series, vol. 3, no. 1, pp. 44-52, 1995.

[8] L.H. Chen, C.C. Hsueh and C.J. Chang, A two stage approach for formulating fuzzy regression models, Knowledge-Based Systems, vol. 52, pp. 302-310, 2013.

[9] I. De and J. Sil, A fuzzy regression analysis based on no reference image quality metric, Advances in Intelligent Informatics, Ed. E.M. El-Alfy, S.M. Thampi, H. Takagi, S. Piramthu and T. Hanne, Advances in Intelligent Systems and Computing, vol. 320, pp. 97-95, 2015.

[10] P. Diamond, Fuzzy Least Squares, Information Sci., vol. 46, pp. 141-157, 1998.

[11] D. Dubois, and H. Prade, Gradualness, uncertainty and bipolarity: Making sense of fuzzy sets, Fuzzy Sets and Systems, vol. 192, no. 1, pp. 3 - 24, 2012.

[12] A.M. Eskicioglu and P.S. Fisher, Image quality measures and their performance, IEEE Trans. Communication, vol. 43, no. 12, pp.2959-2965, 1995.

[13] U. Engelke, A. Maeder and H.J. Zepernick, Human observer confidence in image quality assessment, Signal Processing: Image Communication, vol. 27, pp. 935-947, 2012.

[14] J. Georgii, M. Eder, K. Burger, S. Klotz, F. Ferstl, L. Kovacs and R. Westermann, A computational tool for preoperative breast augmentation planning 
in aesthetic plastic surgery, IEEE Journal of Biomedical and Health Informatics, vol. 18, no. 3, pp. 907-919, 2014.

[15] B. Gladysz and D. Kuchta, A method of variable selection for fuzzy regression - the possibility approach, Operations Research and Decisions, vol. 21, no. 5, pp. 5-15, 2011.

[16] R.Y.K. Fung, Y. Chen and J. Tang, Estimating the functional relationships for quality function deployment under uncertainties, Fuzzy Sets and Systems, vol. 157, pp. 98-120, 2006.

[17] W. Fu, M. Johnston, and M. Zhang, Low-Level Feature Extraction for Edge Detection Using Genetic Programming, IEEE Transactions on Systems, Man and Cybernetics, vol. 44, pp. 1459-1472, 2014.

[18] H. Hassanpour, H.R. Maleki and M.A. Yaghoobi, A goal programming approach to fuzzy linear regression with fuzzy input-output data, Soft Computing, vol. 15 , pp. 1569-1580, 2011

[19] H. Höglund, Fuzzy linear regression-based detection of earnings management, Expert Systems with Applications, vol. 40, pp. 6166-6172, 2013.

[20] T. Hong and P. Wang, Fuzzy interaction regression for short term load forecasting, Fuzzy Optimiz. and Decis. Making, vol. 13, no. 1, pp. 91-103, 2014.

[21] S.C. Horng, S.Y. Lin, L.H. Lee, C.H. Chen, Memetic algorithm for real-time combinatorial stochastic simulation optimization problems with performance analysis, IEEE Transactions on Systems, Man and Cybernetics, vol. 43, no. 5, pp. 1495-1509, 2013.

[22] Y. Hu and P.C. Loizou, Evaluation of objective quality measures for speech enhancement, IEEE Trans. Audio, Speec. Lang. Proc., vol. 16, pp. 229-238, 2008.

[23] C.Y. Huang and G.H. Tzeng, Multiple generation product life cycle predictions using a novel two-stage fuzzy piecewise regression analysis method, Technological Forecasting and Social Change, vol. 75, 12-31, 2008.

[24] H. Jiang, C.K. Kwong, W.H. Ip and Z. Chen, Chaos-based fuzzy regression approach to modeling customer satisfaction for product design, IEEE Transactions on Fuzzy Systems, vol. 21, no. 5, pp. 926-936, 2013.

[25] C. Kao and C.L. Chyu, Least squares estimates in fuzzy regression analysis, European Journal of Operational Research, vol. 148, pp. 426-435, 2003.

[26] U.T. Khan and C. Valeo, A new fuzzy linear regression approach for dissolved oxygen prediction. Hydrological Sciences Journal, vol. 60, no. 6, pp. 1096-1119, 2015 .

[27] K.J. Kim, H. Moskowitz and M. Koksalan, Fuzzy versus statistical linear regression, European Journal of Operational Research, vol. 92, pp. 417-434, 1996.

[28] D.G. Kleinbaum and L.L. Kupper, Applied regression analysis and other multivariable methods, Duxbury Press, 1978.

[29] P. Koutsabasis and T.G. Istikopoulou, Perceived website aesthetics by users and designers: implications for evaluation practice, International Journal of Technology and Human Interaction, vol. 9, no. 2, pp. 39-52, 2013.

[30] J. Koza, Genetic Programming as a means for programming computers by natural selection, Statistics and Computing, vol. 4, pp. 87-112, 1994.

[31] J.R. Koza, Human-competitive results produced by genetic programming, Genetic Programming and Evolvable Machines, vol. 11, pp. 251-294, 2010.

[32] A.O. Laplume and M.K. Srivastava, Firm newness, product novelty and aesthetic failure, Management Decision, vol. 52, no. 10, pp. 1952-1967, 2014.

[33] R. Likert, A technique for the measurement of attitudes, Archives of Psychology, vol. 22, no. 140, pp. 55, 1932.

[34] Z. Geng, J. Chen, and Y. Han, Energy efficiency prediction based on PCAFRBF model: a case study of ethylene industries, IEEE Transactions on Systems, Man and Cybernetics - Systems, 2016.

[35] M. Prasad, C.T. Lin, D.L. Li, C.T. Hong, W.P. Ding, and J.Y. Chang, Softboosted self-constructing neural fuzzy inference network, IEEE Transactions on Systems, Man and Cybernetics - Systems, 2016.

[36] Y. Yu, T.M. Choi, C.L. Hui, and T.K. Ho, A new and efficient intelligent collaboration scheme for fashion design, IEEE Transactions on Systems, Man and Cybernetics - Systems, vol. 41, no. 3, pp. 463-475, 2011.

[37] X. Liu and Y. Chen, A systematic approach to optimizing h value for fuzzy linear regression with symmetric triangular fuzzy numbers, Mathematical Problems in Engineering, Article ID 210164, pp. 1-9, 2013.

[38] Y. Liu, Y. Chen, J. Zhou and S. Zhong, Fuzzy linear regression models for QFD using optimized $h$ values, Eng. App. Artificial Intelligence, 2016. (in press).

[39] J. Lu and R Wang, An enhanced fuzzy linear regression model with more flexible spreads, Fuzzy Sets and Systems, vol. 160, 2505-2523, 2009.

[40] J. Madar, J. Abonyi and F. Szeifert, Genetic programming for the identification of nonlinear input - output models, Industrial and Engineering Chemistry Research, vol. 44, pp. 3178-3186, 2005.

[41] L. Marchesotti, N. Murray and F. Peronnin, Discovering beautiful attributes for aesthetic image analysis, Inter. Jour. Comput. Vision, vol. 113, pp. 246-266, 2015.

[42] M.H. Mashinchi, M.A. Orgun, and M. Mashinchi, Solving fuzzy linear regression with hybrid optimization, Proceedings of the International Conference on Neural Information Processing, pp. 336-343, 2009.

[43] G. Mauris, L. Berrah, L. Foulloy, and A. Haurat, Fuzzy handling of measurement errors in instrumentation, IEEE Transactions on Instrumentation and Measurement, vol. 49, no. 1, pp.89-93, 2000.
[44] W.H. Mormann, B. Stawarczyk, A. Ender, B. Sener, T. Attin and A. Mehl, Wear characteristics of current aesthetic dental restorative CAD/CAM materials: two-body wear, gloss retention, roughness and Martens hardness, Journal of The Mechanical Behavior of Biomedical Materials, vol. 20, pp. 113-125, 2013.

[45] S. Muzzioli, A. Ruggieri and B. De Baets, A comparison of fuzzy regression methods for the estimation of the implied volatility smile function, Fuzzy Sets and Systems, vol. 266, no. 1, pp. 131-143, 2015.

[46] M.M.Nasrabadi and E.Nasrabadi, A mathematical-programming approach to fuzzy linear regression analysis, Applied Mathematics and Computation, vol. 155, no. 3, pp. 873-881, 2004.

[47] T. Niimura and T. Nakashima, Deregulated electricity market data representation by fuzzy regression models, IEEE Trans. Systems, Man and Cybernetics, vol. 31, no. 3, pp. 320-326, 2001.

[48] D.A. Norman, Emotion and Attractive, Interactions, Aug., pp. 36- 42, 2002.

[49] O. Poleshchuk and E. Komarrov, Hybrid fuzzy least square regression model for qualitative characteristics, Integrated Uncertainty Management and Applications, pp. 187-196, 2010.

[50] N. Ponomarenko, L. Jin, O. Ieremeiev, V. Lukin, K. Egiazarian, J. Astola, B. Vozel, K. Chehdi, M. Carli, F. Battisti, C.C.J. Kuo, Image database TID2013: Peculiarities, results and perspectives, Signal Processing: Image Communication, vol. 30, pp. 57-77, 2015

[51] P.P. San, S.H. Ling, Nuryani, H.T. Nguyen, Evolvable Rough-Block-Based Neural Network and its Biomedical Application to Hypoglycemia Detection System. IEEE Transactions on Systems, Man and Cybernetics, vol. 44, no. 8, pp. 1338-1349, 2014

[52] M. Seckler, K. Opwis and A.N. Tuch, Linking objective design factors with subjective aesthetics: an experimental study on how structure and color of websites affect the facets of users' visual aesthetic perception, Computers in Human Behavior, vol. 49, pp. 375-389, 2015.

[53] G. Sekkeli, G. Koksal, I. Batmaz and O.T. Bayrak, Classification models based on Tanaka's fuzzy linear regression approach: The case of customer satisfaction modeling, Journal of Intelligent \& Fuzzy Systems, vol. 21, pp. 341-351, 2010.

[54] Z. Sener and E.E. Karsak, A combined fuzzy linear regression and fuzzy multiple objective programming approach for setting target levels in quality function deployment, Expert Syst. Applications, vol. 38, pp. 3015-3022, 2011.

[55] H. Shakouri and R. Nadimi Outlier detection in fuzzy linear regression with crisp input-output by linguistic variable view, Applied Soft Computing, vol. 13, pp. 734-742, 2013.

[56] Y. Shimizu and Y. Jindo, A fuzzy logic analysis method for evaluation human sensitivities, Inter. Jour. of Industrial Ergonomics, vol. 15, pp. 39-47, 1995.

[57] H. Tanaka, S. Uejima and K. Asai, Linear regression analysis with fuzzy model, IEEE Transactions on Systems, Man and Cybernetics, vol. 12, pp. 903-907, 1982.

[58] J. Tarvainen, M. Sjoberg, S. Westman, J. Laaksonen and P. Oittinen, Contentbased prediction of movie style, aesthetics, and affect: data set and baseline experiments, IEEE Trans. Multimedia, vol. 16, no. 8, pp. 2085-2098, 2014.

[59] M.T. Tsai, K.A. Wang, Y. Liu and J.S. Hong, Perceived visual aesthetics of text-overlaid images: computational models and experimental research for whitespace fraction, Inter. Jour. Human Computer Interaction, vol. 29, pp. 1-23, 2014.

[60] J.V. Tu, Advantages and disadvantages of using artificial neural networks versus logistic regression for predicting medical outcomes, Journal of Clinical Epidemiology, vol. 49, no. 11, pp. 1225-1231, 1996.

[61] P.D. Urso, and T. Gastaldi, An "orderwise" polynomial regression procedure for fuzzy data, Fuzzy sets and Systems, vol. 130, pp. 1-9, 2002.

[62] P.D. Urso, Linear regression analysis for fuzzy/crisp input and fuzzy/crisp output data, Computational Statist. Data Analy., vol. 42, no. 1-2, pp. 47-72, 2003.

[63] H.F. Wang and R.C. Tsaur, Insight of fuzzy regression model, Fuzzy Sets and Systems, vol. 112, pp. 355-369, 2000.

[64] J. Watada, S. Wang and W. Pedrycz, Building confidence-interval-based fuzzy random regression models, IEEE Trans. Fuzzy Sys., vol. 17, pp. 1273-1283, 2009.

[65] T. Weise and K. Tang, Evolving distributed algorithms with genetic programming, IEEE Trans. Evolutionary Comp., vol. 16, no. 2, pp. 242-265, 2012.

[66] Y.M. Wi, S.K. Joo and K.B. Song, Holiday load forecasting using fuzzy polynomial regression with weather feature selection and adjustment, IEEE Transactions on Power Systems, vol. 27, no. 2, pp. 596-603, 2012.

[67] J. Wu, W. Lin, G. Shi, and A. Liu, Reduced-reference image quality assessment with visual information fidelity, IEEE Transactions on Multimedia, vol. 15, no. 7, pp. 1700-1705, 2013.

[68] L.A. Zadeh, The concept of a Linguistic variable and its application to approximate reasoning - I, Information Sciences, vol. 8, pp. 199-249, 1975.

[69] A. Zaric, N. Tatalovic, N. Brajkovic, H. Hlevnjak, M. Loncaric, E. Dumic and S. Grgic, VCL@FER Image Quality Assessment Database, Automatika, vol. 53, no. 4, pp. 344-354, 2012.

[70] P. Zhang and N. Li, The importance of Affective Quality, Communications of the ACM, vol. 48, no. 9, pp. 105-108, 2005.

[71] M. Zhang, D. Wang, Z. Wang, and X. Zhang, Assessing the aesthetic value of urban forests and associated individual differences: an example from the Loess Plateau, Advanced Science and Technology Letters, vol. 49, pp. 274-280, 2014. 


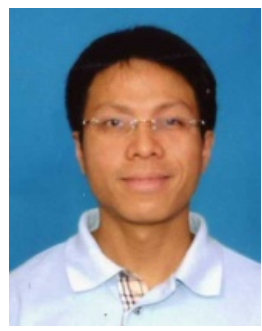

Kit Yan Chan received a $\mathrm{PhD}$ degree in Computing from London South Bank University, United Kingdom, in 2006. He is currently a Senior Lecturer in the Department of Electrical and Computer Engineering, Curtin University, Australia. He was the guest editor for Applied Soft Computing, Neurocomputing, Engineering Applications of Artificial Intelligence, International Journal of Fuzzy Systems (IJFS) and Journal of Engineering Design (JED). He is the associate editor for Neurocomputing and IJFS, and the editorial board of JED. His research interests include machine learning applications.

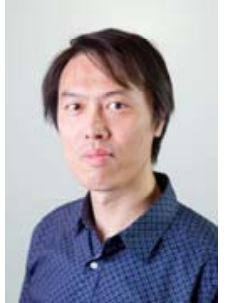

Hak-Keung Lam (M'98-SM'10) received the B.Eng. (Hons.) and Ph.D. degrees from the Department of Electronic and Information Engineering, The Hong Kong Polytechnic University, Hong Kong, in 1995 and 2000, respectively. He joined as a Lecturer at King's College London in 2005 and is currently a Reader. His current research interests include intelligent control systems and computational intelligence. $\mathrm{He}$ is an associate editor for a number of international journals and is an IEEE senior member.

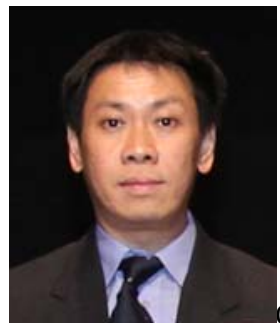

Cedric Ka Fai Yiu received his M.Sc. from University of Dundee and University of London, and D.Phil. from University of Oxford. He is currently working in the Hong Kong Polytechnic University. He has published over 100 journal publications and given over 30 conference presentations. He holds two U.S. patents in signal processing. He received the third prize of Chongqing Natural Science Foundation Award in 2014. His current research interests include optimization and optimal control, signal processing and financial risk management.

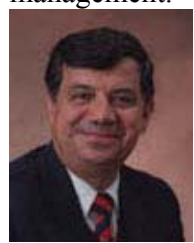

Tharam Dillon (M'74-SM'87-F'98-LF'10) is a Fellow of the IEEE, ACS and IE(Aust). He is the professor at Department of Computer Science and Computer Engineering, La Trobe University, Australia. He has published more than 750 papers published in international conferences and journals and is the author of 5 books and has another 5 edited books. His current research interests include Web semantics, ontologies, Internet computing, e-commerce, hy-brid neurosymbolic systems, neural nets, software engineering, database systems, and data mining. He is the Editor-in-Chief of the International Journal of Computer Systems Science and Engineering as well as the Engineering Intelligent Systems. 